\title{
Virginia On-Farm Soybean Research
}

A summary of replicated research conducted by

Virginia Cooperative Extension in cooperation with local producers and agribusiness
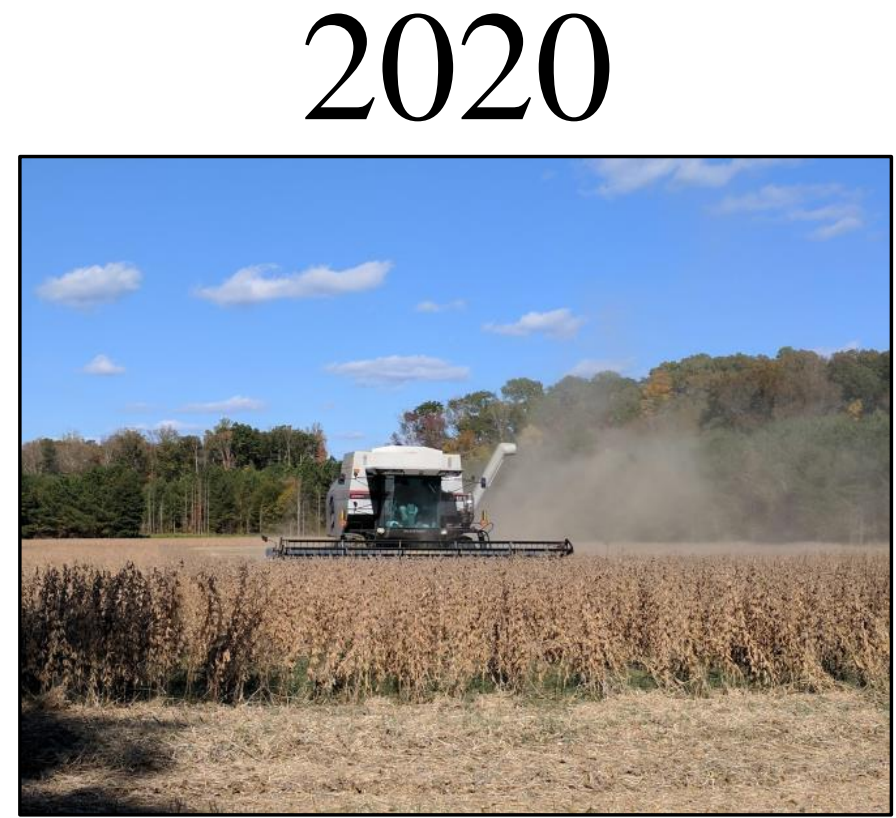

Conducted and Summarized by the following Extension Faculty:

Scott Reiter, Prince George County

Stephanie Romelczyk, Westmoreland County

Mike Broaddus, Caroline/King George Counties

Taylor Clarke, Mecklenburg County

Lindy Fimon, Lunenburg County

Roy Flanagan, City of Virginia Beach

Josh Holland, Southampton County

Bruce Jones, Appomattox County

Joanne Jones, Charlotte County

Trent Jones, Lancaster/Northumberland Counties

Watson Lawrence, City of Chesapeake

Robbie Longest, Essex County

Mike Parrish, Dinwiddie County

Sara Rutherford, Greensville County/City of Emporia

Carl Stafford, Culpeper County

David Holshouser, Virginia Tech-Tidewater AREC 


\section{Introduction}

These results are a collaborative effort of Virginia Cooperative Extension (VCE) Agents and Specialists, area producers, and agribusiness. The purpose of this publication is to provide research-based information to aid in the decision-making process for soybean producers in Virginia. It provides an unbiased evaluation of varieties, management practices, and new technologies through on-farm replicated research using producer equipment and time. These experiments enable producers to make better management decisions based on research and provide greater opportunities to improve yields and profits, which improves quality of life for them and their families.

The success of these on-farm experiments is very dependent on the cooperative effort of the producer and the assisting agribusinesses. We are grateful for that cooperation. We hope the information will be beneficial to you and your individual agribusiness operations. This publication is made available each year at the Virginia Grain and Soybean Conference, at regional production meetings throughout Virginia, and on the VCE website (http://resources.ext.vt.edu). This information reaches hundreds of Virginia soybean and grain producers plus agribusinesses, impacting over 550,000 acres of soybeans valued at approximately \$200 million.

The field work and printing of this publication is supported by Virginia Soybean Board Check-Off Funds. The cooperators graciously wish to acknowledge this support. Any producer or agribusiness professional wishing to receive a copy of this publication should contact their local Extension Agent who can request a copy from Stephanie Romelczyk in Westmoreland County at 804-493-8924 or sromelcz@ vt.edu.

This is the 24th year of this multi-county cooperative effort and further work is planned for 2021. The authors wish to thank the many producers who participated in this project. Appreciation is extended to seed, crop protection, and fertilizer representatives who donated products and/or assisted with the field work.

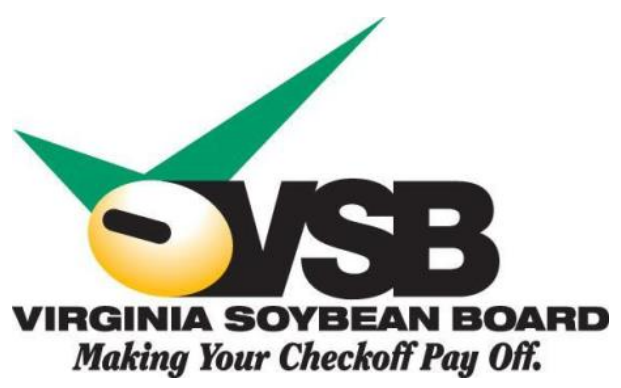

DISCLAIMER: Trade and brand names are used only for educational purposes, and Virginia Cooperative Extension does not guarantee or warrant the standards of the product, nor does Virginia Cooperative Extension imply approval of the product to the exclusion of others which may also be suitable. 


\section{Table of Contents}

General Summary

$\begin{array}{ll}\text { Trait Data for On-Farm Soybean Variety Tests } & 5\end{array}$

$\begin{array}{ll}\text { Soybean Herbicide Systems and Herbicide Selection Chart } & 7\end{array}$

$\begin{array}{lr}\text { Seed Treatment Data for On-Farm Soybean Variety Tests } & 8\end{array}$

Maturity Group 4 Variety Comparisons 9

2020 Overall Group 4 Comparison $\quad 10$

$\begin{array}{ll}\text { Caroline } & 11\end{array}$

Chesapeake/Virginia Beach $\quad 12$

$\begin{array}{ll}\text { Culpeper } & 13\end{array}$

$\begin{array}{ll}\text { Mecklenburg } & 14\end{array}$

Northumberland Ag Expo $\quad 16$

$\begin{array}{ll}\text { Prince George } & 18\end{array}$

Southampton $\quad 20$

Westmoreland 21

Maturity Group 5 Variety Comparisons $\quad 23$

2020 Overall Group 5 Comparison $\quad 24$

Brunswick $\quad 25$

$\begin{array}{ll}\text { Charlotte } & 27\end{array}$

Dinwiddie $\quad 28$

Northumberland Ag Expo $\quad 30$

Prince George $\quad 32$

Southampton $\quad 33$

$\begin{array}{ll}\text { Other Soybean Weed Control System Tests } & 34\end{array}$

2020 Overall LibertyLink Comparison $\quad 35$

$\begin{array}{ll}\text { Brunswick } & 36\end{array}$

$\begin{array}{ll}\text { Other Research } & 38\end{array}$

Northumberland Ag Expo Maturity Group 2.0 - 3.9 Soybean Comparison 39

Westmoreland Soybean Following Cover Crop Study $\quad 40$

Essex Plenish Soybean Evaluation Study

Essex Brassica Cover Crop Soybean Demonstration 43

Northumberland Double-Crop Soybean Seeding Rate Study 46

Northumberland Full-Season Soybean Seeding Rate Study $\quad 47$

Northumberland Ag Expo Full-Season Soybean Seeding Rate Study 48

Suffolk Late-Planted Soybean Seeding Rate Study 49

PHOTOS: Courtesy of Lindy Fimon, Laura Siegle, Scott Reiter, Trent Jones, Robbie Longest, and Stephanie Romelczyk 


\section{GENERAL SUMMARY}

First, we would like to thank everyone that participated in on-farm plot work: seed and input suppliers for providing materials for the trials, our farmer-cooperators for supplying equipment, land, and patience to get these tests from planting to harvest, the Virginia Soybean Board for funding to assist with expenses, Extension Agents for securing locations, hauling seed, and sending in data, and you, the soybean grower, for showing interest in our work and taking time to review this publication.

Weather continued to keep us guessing and frustrated in 2020. May and June brought various levels of rain and cool temperatures across the State. Then hot, dry conditions set in July for about 3 weeks. August and September provided record rainfall for much of Virginia. Overall, yields have been very good across trial locations. Harvest was also a struggle with wet soils and high moisture seed for much of the season. Weather is still a risk difficult to manage.

As in the past, Extension Agents have compared Maturity Group (MG) 4 \& 5 varieties across multiple locations. This work is performed in concert with the Official Variety Tests conducted by Dr. David Holshouser and offers producers even stronger yield comparison information that they can use when making planting decisions. In addition, a special MG 2.5-3.9 trial was conducted at the Virginia Ag Expo site in Northumberland County.

For 2020, the decision was made to accept only Roundup Ready 2 Xtend soybeans for the Roundup Ready trials. This simplified management for grower cooperators and eliminated damage to non-Xtend plots. This also represented the current trend in new soybean variety offerings. The LibertyLink trials included LibertyLink, LibertyLink GT27, and Enlist E3 varieties. Included in this publication is a chart with the various herbicide systems and corresponding herbicides. Weed control system, nematode resistance, and disease package should be considered when selecting varieties for 2021.

The widespread use of cover crops and a focus on soil health continue to look at yield advantage and return on investment. A study in Westmoreland evaluated wheat, barley, oats, and rye cover crop effects on biomass and soybean yields. Another study in Essex investigated the effect of brassica cover crops on in-season nutrient cycling.

A demonstration of Plenish soybeans was planted to evaluate yield. Plenish soybeans produce a high oleic oil that is desired by some processors. Some Virginia soybean buyers have programs for Plenish soybeans. Four seeding rate trials were conducted in full-season and double-crop plantings. The 2020 results continue to reinforce that yields can be maintained with April- or May-planted seeding rates of 90,000-125,000 seed/acre and 160,000200,000 seed/acre with late plantings.

We hope you find this information useful. If you have ideas for 2021 on-farm research or would like to be a cooperator in 2021, please contact your local Virginia Cooperative Extension Agriculture Agent. 


\section{Trait Data for 2020 VCE On-farm Soybean Varieties}

\section{Roundup Ready 2 Xtend}

\begin{tabular}{|c|c|c|c|c|c|c|c|c|c|}
\hline Company & Brand & $\frac{\text { Relative }}{\text { Maturity }}$ & $\frac{\text { Herbicide }}{\text { Traits }}$ & $\frac{\text { Soybean Cyst }}{\text { Nematode }}$ & $\frac{\text { Root Knot }}{\text { Nematode }}$ & $\frac{\text { Frogeve }}{\text { leafspot }}$ & $\frac{\text { Sudden death }}{\text { syndrome }}$ & $\frac{\text { Brown }}{\text { stem rot }}$ & $\frac{\text { Cercospora }}{\text { blight }}$ \\
\hline$\overline{\text { Asgrow }}$ & $\overline{A G 47 \times 9}$ & 4.7 & RR2X & $\mathrm{R} 3$ & $S$ & $\overline{V G}$ & & & \\
\hline Asgrow & AG48X9 & 4.8 & RR2X/SR & R3 & $\mathrm{S}$ & G & VG & & \\
\hline Credenz & CZ 4869X & 4.8 & RR2X & G & $\mathrm{S}$ & VG & G & & \\
\hline Credenz & CZ 4979X & 4.9 & RR2X & & $\mathrm{F}$ & G & G & & \\
\hline Dyna-Gro & S48XT56 & 4.8 & RR2X & R3, MR14 & $\mathrm{S}$ & VG & VG & & VG \\
\hline Dyna-Gro & S48XT90 & 4.8 & RR2X & S & $\mathrm{F}$ & VG & VG & & \\
\hline Hubner & H46-29R2X & 4.6 & RR2X/SR & R3 & S & $\mathrm{F}$ & G & & \\
\hline Hubner & H49-27R2X & 4.9 & RR2X/SR & MR1, R3 & $\mathrm{s}$ & G & G & & \\
\hline LG Seed & C4845RX & 4.8 & RR2X & R3, MR14 & MR & VG & VG & & VG \\
\hline LG Seed & C4227RX & 4.2 & RR2X/STS & R3, MR14 & $\mathrm{S}$ & G & $\mathrm{E}$ & & \\
\hline Local Seed Company & LS4889XS & 4.8 & RR2X/STS & R3, MR14 & $\mathrm{S}$ & G & G & & \\
\hline Local Seed Company & LS4999X & 4.9 & RR2X & R3, MR14 & $\mathrm{S}$ & $\mathrm{E}$ & VG & & \\
\hline MorSoy & MS 4616 RXT & 4.6 & RR2X/STS & MR & $\mathrm{S}$ & $\mathrm{E}$ & G & & \\
\hline MorSoy & MS 4846 RXT & 4.8 & RR2X & MR & $\mathrm{S}$ & VG & VG & & \\
\hline NK Seed & S42-B9XS & 4.2 & RR2X/STS & R3 & $\mathrm{S}$ & G & VG & VG & \\
\hline NK Seed & S44-C7X & 4.4 & RR2X & $\mathrm{R} 3,14$ & G & VG & G & & \\
\hline Pioneer & P42A96X & 4.2 & RR2X & $\mathrm{R}$ & $\mathrm{S}$ & VG & VG & & \\
\hline Pioneer & P48A60X & 4.8 & RR2X & R & S & $\mathrm{F}$ & VG & & \\
\hline Progeny & $4821 \mathrm{RX}$ & 4.8 & RR2X & R3, MR14 & $\mathrm{S}$ & MR & MR & & \\
\hline USG & 7447XTS & 4.4 & RR2X/STS & R3, MR14 & $\mathrm{S}$ & MR & MR & & \\
\hline USG & $7480 X T$ & 4.8 & RR2X & S & MS & MR & MR & & \\
\hline Asgrow & AG55X7 & 5.5 & RR2X & $\mathrm{s}$ & $\mathrm{R}$ & G & G & & \\
\hline Asgrow & AG56X8 & 5.6 & RR2X & $\mathrm{R} 1,3$ & $\mathrm{R}$ & VG & G & & \\
\hline Credenz & CZ 5299X & 5.2 & RR2X & & S & G & G & & \\
\hline Credenz & CZ 5420X & 5.4 & RR2X & & $\mathrm{S}$ & G & G & & \\
\hline Dyna-Gro & S56XT99 & 5.6 & RR2X & $\mathrm{R} 1,3$ & VG & VG & G & & VG \\
\hline Hubner & H50-10R2X & 5.0 & RR2X/SR & R3 & $S$ & & VG & & \\
\hline LG Seed & LGS5315RX & 5.3 & RR2X & R3, MR13 & & VG & VG & & \\
\hline Local Seed Company & LS5087X & 5.0 & RR2X & R3, MR14 & $\mathrm{S}$ & EX & VG & & \\
\hline Local Seed Company & LS5386X & 5.3 & RR2X & R3, MR14 & MR-MS & VG & G & & \\
\hline MorSoy & MS 5398 RXT & 5.3 & RR2X & MR & S & G & G & & \\
\hline MorSoy & MS 5607 RXT & 5.6 & RR2X & MR & $\mathrm{E}$ & EX & G & & \\
\hline NK Seed & S51-R3XS & 5.1 & RR2X/STS & R3, MR14 & $\mathrm{F}$ & VG & G & & \\
\hline NK Seed & S53-F7X & 5.3 & RR2X & MR3, R14 & $\mathrm{F}$ & VG & VG & & \\
\hline Pioneer & P52A05X & 5.2 & RR2X & R & E & G & G & & \\
\hline Pioneer & P55A49X & 5.5 & RR2X & $\mathrm{R}$ & $\mathrm{E}$ & G & G & & \\
\hline Progeny & $5016 \mathrm{RXS}$ & 5.0 & RR2X/STS & R3, MR14 & MR & MR & MR & & \\
\hline USG & 7529XTS & 5.2 & RR2X/STS & S & $\mathrm{S}$ & MR & MS & & \\
\hline USG & $7540 X \mathrm{~T}$ & 5.4 & RR2X & S & S & MR & MR & & \\
\hline \multirow{2}{*}{\multicolumn{10}{|c|}{\begin{tabular}{l|l}
$R=$ Resistant & No entry for a particular trait means that no information was \\
$S=$ Susceptible & provided or trait has not been rated by the company. \\
$M R$ & $=$ Moderately resistant \\
$M=$ Moderate &
\end{tabular}}} \\
\hline & & & & & & & & & \\
\hline \multicolumn{5}{|c|}{$\begin{array}{l}\text { RR2X }=\text { Roundup Ready } 2 \text { Xtend } \\
\text { STS or SR }=\text { Tolerant to sulfonylurea herbicides }\end{array}$} & \multicolumn{5}{|c|}{$\begin{array}{c}\text { All ratings were taken from company literature available in } \\
\text { current catalogs or websites. }\end{array}$} \\
\hline
\end{tabular}




\section{Trait Data for 2020 VCE On-farm Soybean Varieties}

\section{LibertyLink, LibertyLink GT27, Enlist E3, and Early Roundup Ready 2 Xtend}

\begin{tabular}{|c|c|c|c|c|c|c|c|c|c|}
\hline Company & Brand & $\frac{\text { Relative }}{\text { Maturity }}$ & $\underline{\text { Herbicide Traits }}$ & $\frac{\text { Soybean Cyst }}{\text { Nematode }}$ & $\frac{\text { Root Knot }}{\text { Nematode }}$ & $\frac{\text { Frogeye }}{\text { leafspot }}$ & $\frac{\text { Sudden death }}{\text { syndrome }}$ & $\frac{\text { Brown }}{\text { stem rot }}$ & $\frac{\text { Cercospora }}{\text { blight }}$ \\
\hline Credenz & CZ 4539 GTLL & 4.5 & LLGT27 & VG & S & VG & G & VG & \\
\hline Dyna-Gro & S49EN79 & 4.9 & E3 & R3, MR14 & $\mathrm{S}$ & VG & G & & \\
\hline Local Seed Company & LS4706GL & 4.7 & LLGT27 & R3, MR14 & $S$ & VG & VG & & \\
\hline Local Seed Company & ZS4694E3S & 4.6 & E3/STS & R3, MR14 & $S$ & $E$ & & & \\
\hline Credenz & CZ 5147 LL & 5.1 & $\mathrm{LL}$ & & MR & $E$ & $E$ & $E$ & \\
\hline Credenz & CZ 5859 LL & 5.8 & LL & MR & MR & VG & & & \\
\hline Dyna-Gro & S52LL66 & 5.2 & LL & MR3 & $\mathrm{F}$ & VG & G & & \\
\hline Dyna-Gro & S55LS75 & 5.5 & LL/STS & $S$ & $\mathrm{~F}$ & VG & $\mathrm{G}$ & & \\
\hline Asgrow & AG36X6 & 3.6 & RR2X & R3 & $\mathrm{S}$ & $\mathrm{F}$ & G & VG & \\
\hline Asgrow & AG38X8 & 3.8 & RR2X & R3 & S & VG & G & & \\
\hline Channel & 3919R2X & 3.9 & RR2X & $\mathrm{R}$ & & $\mathrm{F}$ & & & \\
\hline Dyna-Gro & S37XS89 & 3.7 & RR2X/STS & R3, MR14 & & VG & $\mathrm{G}$ & & \\
\hline LG Seed & LGS3777RX & 3.7 & RR2X & R3, MR14 & & VG & VG & $\mathrm{E}$ & \\
\hline LG Seed & C2888RX & 2.8 & RR2X & R3, MR14 & & & VG & $E$ & \\
\hline Local Seed Company & LS3976X & 3.9 & RR2X & R3, MR14 & MS & $E$ & $\mathrm{G}$ & & \\
\hline MorSoy & MS 3907 RXT & 3.9 & RR2X & MR & $\mathrm{S}$ & $\mathrm{F}$ & G & & \\
\hline NK Seed & S39-G2X & 3.9 & RR2X & R3, R14 & $\mathrm{F}$ & G & VG & & \\
\hline NK Seed & S37-A4X & 3.7 & RR2X & R3, MR14 & $\mathrm{F}$ & $\mathrm{G}$ & VG & & \\
\hline Pioneer & P39A58X & 3.9 & RR2X & R3, MR14 & $S$ & VG & G & & \\
\hline
\end{tabular}

$\mathrm{R}=$ Resistant

$\mathrm{S}=$ Susceptible

$\mathrm{MR}=$ Moderately resistant

$\mathrm{M}=$ Moderate

MS $=$ Moderately susceptible

RR2X = Roundup Ready 2 Xtend

$\mathrm{E} 3=$ Enlist E3

$\mathrm{LL}=$ LibertyLink

LLGT27 = LibertyLink GT27

STS or SR $=$ Tolerant to sulfonylurea herbicides
No entry for a particular trait means that no information was provided or trait has not been rated by the company.

All ratings were taken from company literature available in current catalogs or websites. 


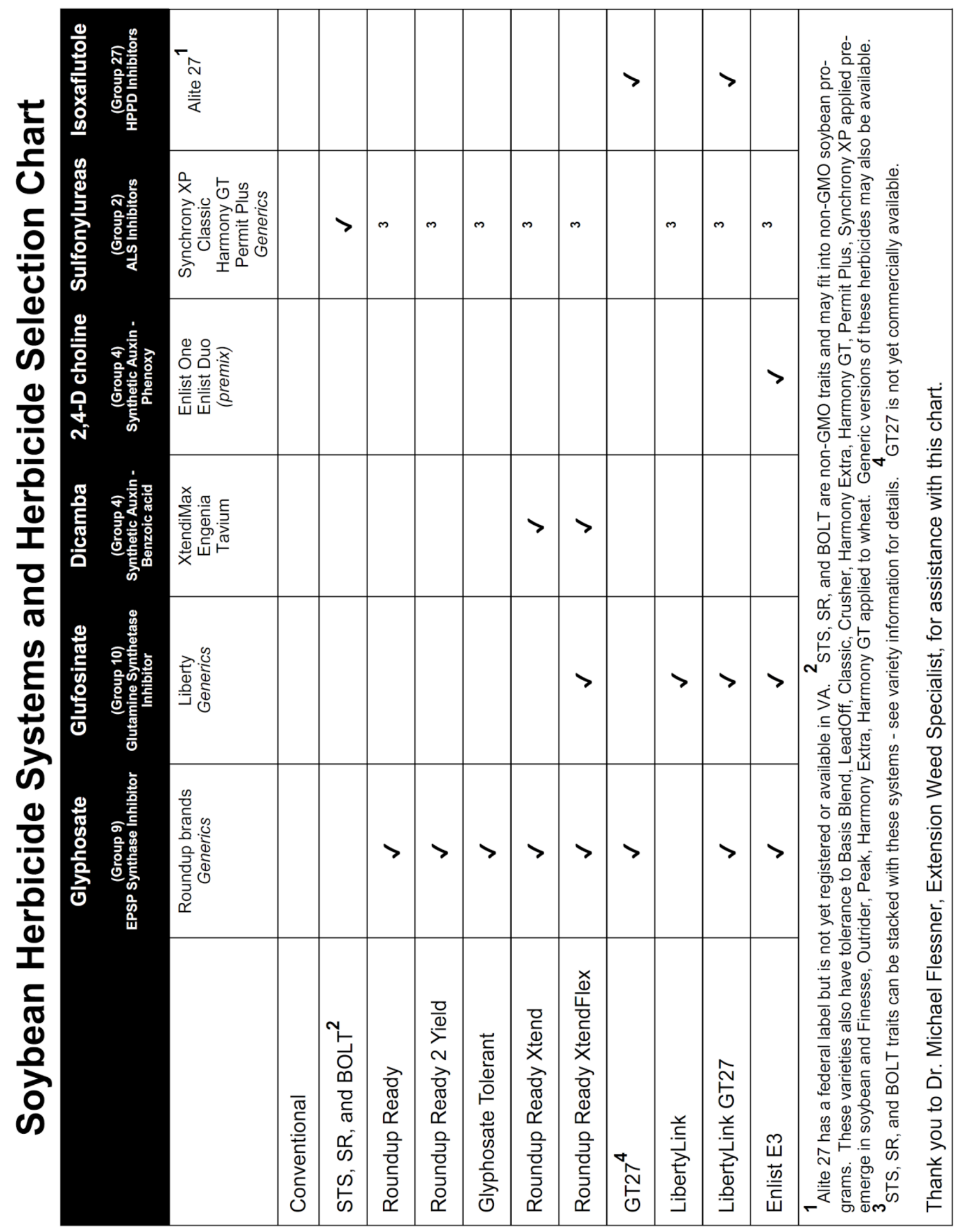




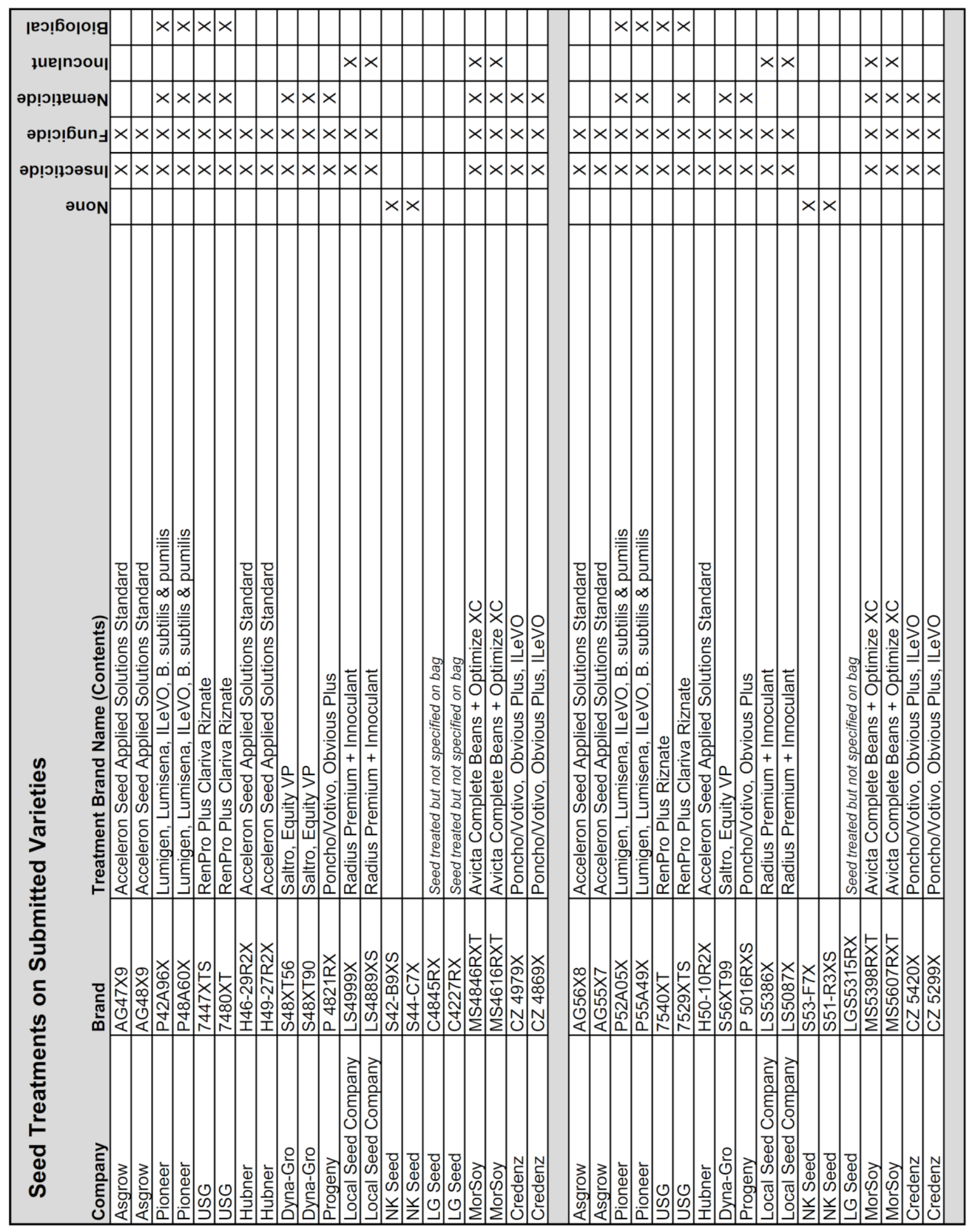




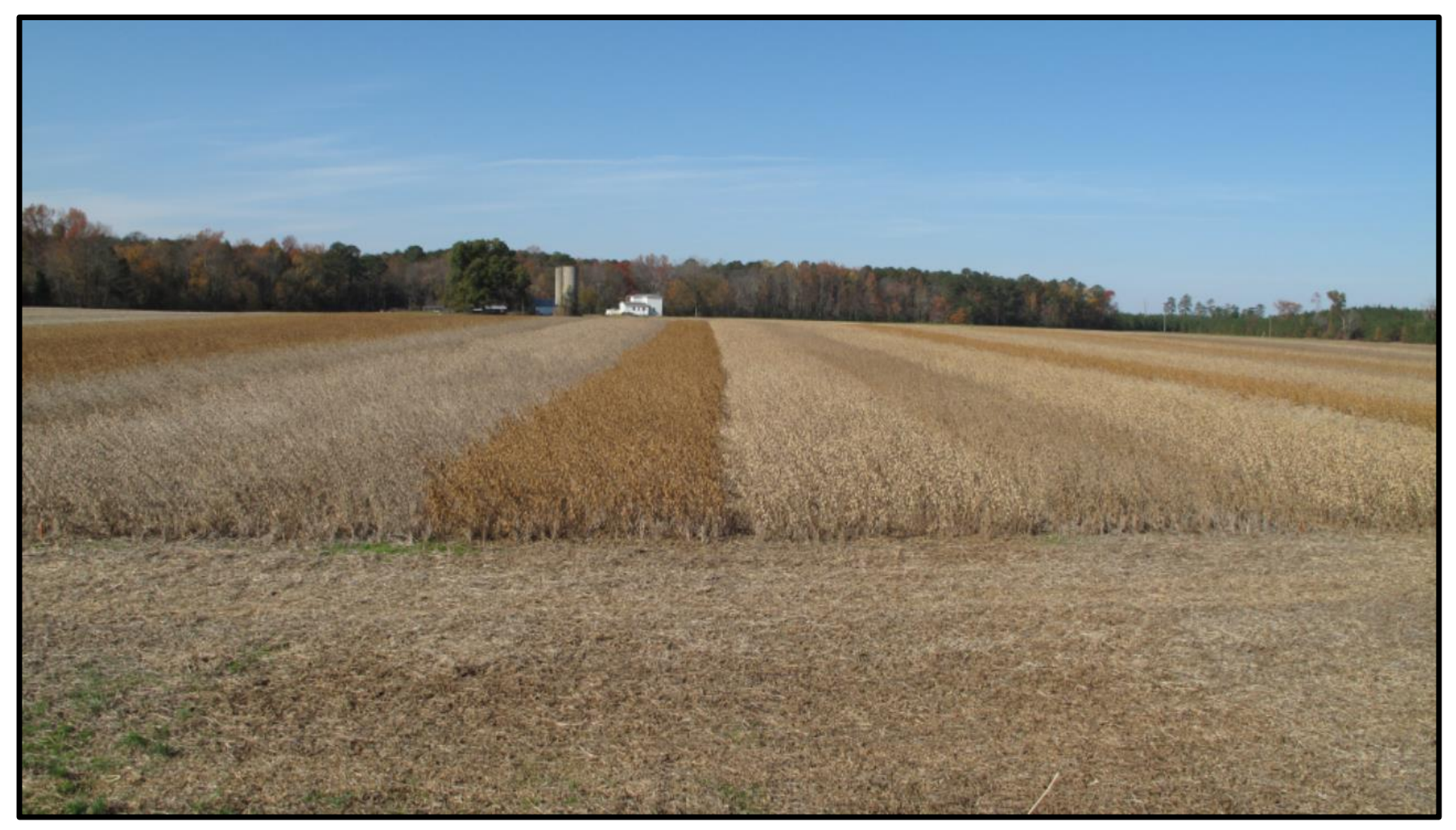

\section{MATURITY GROUP 4 VARIETY COMPARISONS}




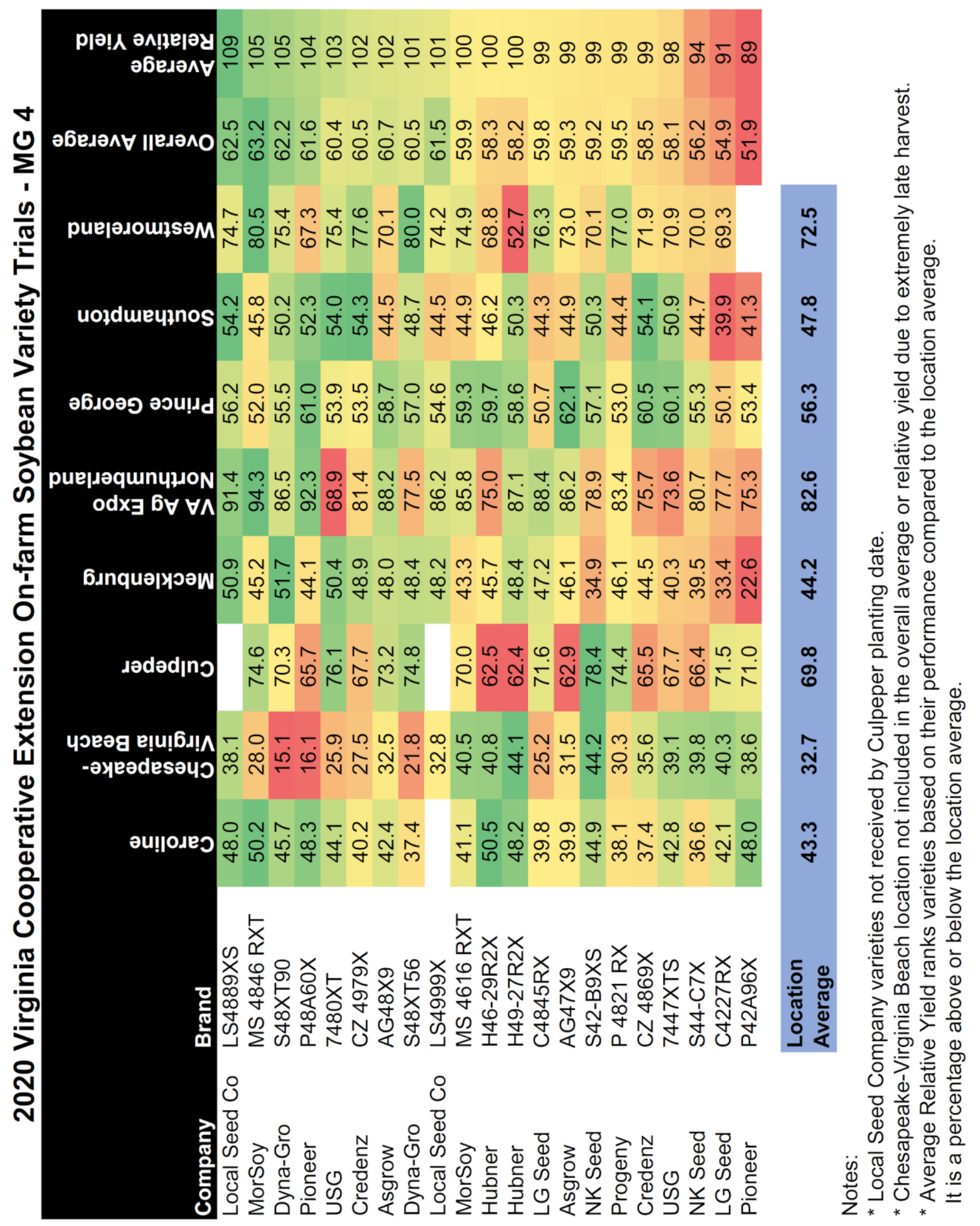




\section{CAROLINE COUNTY MATURITY GROUP 4 SOYBEAN COMPARISONS}

Cooperators:

Previous Crop:

Soil Type:

Tillage:

Planting Date:

Seeding Rate/Row Spacing: 175,000 seeds/A drilled in 7-inch rows

Fertilization:

none

Crop Protection: Burndown: 1 qt./A PowerMax (glyphosate); 48 oz./A Warrant; 1 pt./100 gal Liberate surfactant.

Harvest Date: December 1, 2020

Harvest Equipment: $\quad$ New Holland CR9040 w/ 30-foot flex head

\begin{tabular}{|l|l|c|c|}
\hline Brand & Variety & Moisture \% & $\begin{array}{c}\text { Yield } \\
\text { (bu./ac.@ 13.0\%) }\end{array}$ \\
\hline USG & 7480XT & 13.0 & 44.1 \\
\hline Pioneer & P42A96X & 12.3 & 48.0 \\
\hline Pioneer & P48A60X & 12.4 & 48.3 \\
\hline Asgrow & AG48X9 & 13.3 & 42.4 \\
\hline Asgrow & AG47X9 & 12.8 & 39.9 \\
\hline Hubner & H46-29R2X & 12.9 & 50.5 \\
\hline Hubner & H49-27R2X & 13.1 & 48.2 \\
\hline Credenz & CZ 4869X & 12.9 & 37.4 \\
\hline Credenz & CZ 4979X & 13.6 & 40.2 \\
\hline LG Seed & C4845RX & 12.7 & 39.8 \\
\hline LG Seed & C4227RX & 12.0 & 42.1 \\
\hline Local Seed Company & LS4889XS & 12.7 & 48.0 \\
\hline Dyna-Gro & S48XT90 & 12.9 & 45.7 \\
\hline Dyna-Gro & S48XT56 & 12.5 & 37.4 \\
\hline MorSoy & MS 4846 RXT & 13.5 & 50.2 \\
\hline MorSoy & MS 4616 RXT & 13.1 & 41.1 \\
\hline NK Seed & S42-B9XS & 14.0 & 44.9 \\
\hline NK Seed & S44-C7X & 13.9 & 36.6 \\
\hline Progeny & P4821RX & 12.8 & 38.1 \\
\hline USG & 7447XTS & 13.1 & 42.8 \\
\hline & AVERAGE & $\mathbf{1 3 . 0}$ & $\mathbf{4 3 . 3}$ \\
\hline
\end{tabular}

Discussion: Planted in standing wheat straw due to a failed wheat crop. Although record heat was recorded in mid-July and early August, this area of Caroline received several rain storms during that time that seemed to produce good double-crop soybean yields. 
2020 CHESAPEAKE/VA BEACH CITY MATURITY GROUP 4 SOYBEAN COMPARISONS

Cooperators:

Producer:

Frank Brickhouse

Extension: Watson Lawrence-Chesapeake VCE

Roy Flanagan-Virginia Beach VCE

Previous Crop:

Corn grain

Soil Type:

Tillage:

Acredale Silt Loam

Planting Date:

Seeding Rate/Row Spacing:

Conventional

June 30, 2020

Fertilization:

Crop Protection:

30-inch rows

500 lbs. 15-15-15/acre

Post-emerg. herbicide: 12 oz. Select +16 . oz. Reflex Insecticide: $9 \mathrm{oz}$. Besiege

Harvest Date:

December 30, 2020

Harvest Equipment:

JD 95 Combine-1967 Model-Operator Trey Brickhouse

\begin{tabular}{|l|l|c|c|}
\hline Brand & Variety & Moisture\% & Yield (bu/A) \\
\hline Pioneer & P42A96X & 13.6 & 38.6 \\
\hline LG Seed & C4227RX & 13.5 & 40.3 \\
\hline NK Seed & S42-B9XS & 13.8 & 44.2 \\
\hline NK Seed & S44-C7X & 13.1 & 39.8 \\
\hline USG & 7447XTS & 13.3 & 39.1 \\
\hline MorSoy & MS 4616 RXT & 13.2 & 40.5 \\
\hline Hubner & H46-29R2X & 13.3 & 40.8 \\
\hline Progeny & P 4821 RX & 13.3 & 30.3 \\
\hline Local Seed Company & LS4889XS & 13.3 & 38.1 \\
\hline USG & 7480XT & 13.5 & 25.9 \\
\hline Pioneer & P48A60X & 13.7 & 16.1 \\
\hline Asgrow & AG48X9 & 13.8 & 32.5 \\
\hline Dyna-Gro & S48XT90 & 13.7 & 15.1 \\
\hline Dyna-Gro & S48XT56 & 13.9 & 21.8 \\
\hline MorSoy & MS 4846 RXT & 13.4 & 28.0 \\
\hline Credenz & CZ 4869X & 13.7 & 35.6 \\
\hline Credenz & CZ 4979X & 13.7 & 27.5 \\
\hline Asgrow & AG47X9 & 13.7 & 31.5 \\
\hline Hubner & H49-27R2X & 13.5 & 44.1 \\
\hline Local Seed Company & LS4999X & 13.8 & 32.8 \\
\hline LG Seed & C4845RX & 13.7 & 25.2 \\
\hline & AVERAGE & $\mathbf{1 3 . 5}$ & $\mathbf{3 2 . 7}$ \\
\hline
\end{tabular}

Discussion: Use these data, as well as other test plot results, when making variety selections. 


\section{CULPEPER COUNTY MATURITY GROUP 4 SOYBEAN COMPARISONS}

Cooperators:

Previous Crop:

Soil Type:

Tillage:

Planting Date:

Seeding Rate/Row Spacing:

Fertilization:

Crop Protection:

Harvest Date:
Producer: $\quad$ The Glebe at Ratrie, Ross Swan

Extension: Carl Stafford, ANR - Culpeper

Industry: John VanderCromert, Hubner

Corn

Fauquier silt loam

No-till

May 27, 2020

$140,000 / 15 "$

80 lbs Ammonium Sulfate, P\&K variable rate removal Glyphosate, Surveil, Salvo, Engenia, Reign

November 4, 2020

\begin{tabular}{|l|l|c|c|}
\hline Brand & Variety & Moisture\% & Yield (bu/A) \\
\hline Check 1 & & 13.9 & 53.6 \\
\hline MorSoy & MS 4616 RXT & 13.8 & 70.0 \\
\hline Progeny & P 4821 RX & 13.0 & 74.4 \\
\hline Credenz & CZ 4869X & 13.4 & 65.5 \\
\hline USG & 7447XTS & 13.4 & 67.7 \\
\hline Credenz & CZ 4979X & 13.5 & 67.7 \\
\hline NK Seed & S42-B9XS & 13.6 & 78.4 \\
\hline MorSoy & MS 4846 RXT & 13.3 & 74.6 \\
\hline Hubner & H46-29R2X & 13.4 & 62.5 \\
\hline USG & 7480XT & 13.2 & 76.1 \\
\hline LG Seed & C4845RX & 13.2 & 71.6 \\
\hline Check 2 & & 13.2 & 57.8 \\
\hline LG Seed & C4227RX & 13.4 & 71.5 \\
\hline Dyna-Gro & S48XT56 & 13.4 & 74.8 \\
\hline Pioneer & P48A60X & 13.1 & 65.7 \\
\hline Hubner & H49-27R2X & 13.0 & 62.4 \\
\hline Dyna-Gro & S48XT90 & 13.0 & 70.3 \\
\hline Asgrow & AG48X9 & 12.7 & 73.2 \\
\hline Asgrow & AG47X9 & 12.8 & 62.9 \\
\hline Pioneer & P42A96X & 13.0 & 71.0 \\
\hline NK Seed & S44-C7X & 13.2 & 66.4 \\
\hline Check 3 & & 13.1 & 51.8 \\
\hline & & & $\mathbf{6 7 . 7}$ \\
\hline & AVERAGE & $\mathbf{1 3 . 3}$ & \\
\hline
\end{tabular}

Discussion: Local Seed Company varieties were not provided to this location at planting. 
2020 MECKLENBURG COUNTY MATURITY GROUP 4 SOYBEAN COMPARISONS

Cooperators:

Previous Crop:

Tillage:

Planting Date:

Seeding Rate/Row Spacing:

Fertilization:

Crop Protection:

Harvest Date:

Harvest Equipment:
Producer:

Extension:

\begin{tabular}{|l|l|c|c|}
\hline Brand & Variety & Moisture\% & Yield (bu/A) \\
\hline CHECK & Axis 4730 & 16.1 & 44.0 \\
\hline Asgrow & AG47X9 & 16.6 & 46.1 \\
\hline Asgrow & AG48X9 & 16.2 & 48.0 \\
\hline Pioneer & P42A96X & 15.8 & 22.6 \\
\hline Pioneer & P48A60X & 16.0 & 44.1 \\
\hline USG & 7447XTS & 15.5 & 40.3 \\
\hline USG & 7480XT & 15.4 & 50.4 \\
\hline Hubner & H46-29R2X & 15.4 & 45.7 \\
\hline Hubner & H49-27R2X & 16.0 & 48.4 \\
\hline CHECK & Axis 4730 & 15.8 & 43.1 \\
\hline Dyna-Gro & S48XT56 & 15.5 & 48.4 \\
\hline Dyna-Gro & S48XT90 & 15.4 & 51.7 \\
\hline Progeny & P 4821 RX & 15.2 & 46.1 \\
\hline NK Seed & S42-B9XS & 15.6 & 34.9 \\
\hline NK Seed & S44-C7X & 15.2 & 39.5 \\
\hline Credenz & CZ 4869X & 15.3 & 44.5 \\
\hline Credenz & CZ 4979X & 15.3 & 48.9 \\
\hline LG Seed & C4845RX & 15.0 & 47.2 \\
\hline CHECK & Axis 4730 & 15.9 & 44.1 \\
\hline LG Seed & C4227RX & 16.0 & 33.4 \\
\hline Local Seed Company & LS4889XS & 16.1 & 50.9 \\
\hline Local Seed Company & LS4999X & 16.2 & 48.2 \\
\hline MorSoy & MS 4616 RXT & 15.0 & 43.3 \\
\hline MorSoy & MS 4846 RXT & 15.1 & 45.2 \\
\hline Dyna-Gro & S49EN79 & 15.9 & 44.6 \\
\hline & & & \\
\hline
\end{tabular}

John Manning

Lindy Fimon, Taylor Clarke

Soybeans

No-till

May 26, 2020

148,000 on 18 " rows

200 lbs 6-15-40

Burndown: Roundup + Envive

POST: Roundup + Flexstar

November 10, 2020

JD 4420 with $15 \mathrm{ft}$ head 


\begin{tabular}{|l|l|c|c|} 
CHECK & Axis 4730 & 15.3 & 48.3 \\
\hline & & & \\
\hline & AVERAGE & $\mathbf{1 5 . 6}$ & $\mathbf{4 4 . 3}$ \\
\hline
\end{tabular}

Discussion: The dry period this summer adversely impacted the earliest varieties. 
2020 NORTHUMBERLAND COUNTY MATURITY GROUP 4 SOYBEAN COMPARISONS

Cooperators:

Previous Crop:

Soil Type:

Tillage:

Planting Date:

Seeding Rate/Row Spacing:

Fertilization:

Crop Protection:

Harvest Date:

Harvest Equipment:

Producer:

Extension:

Industry:

\begin{tabular}{|l|l|c|c|}
\hline Brand & Variety & Moisture\% & Yield (bu/A) \\
\hline MorSoy & MS 4616 RXT & 15.6 & 94.5 \\
\hline MorSoy & MS 4846 RXT & 15.5 & 94.3 \\
\hline Dyna-Gro & S48XT56 & 15.6 & 77.5 \\
\hline Dyna-Gro & S48XT90 & 15.2 & 86.5 \\
\hline Local Seed Company & LS4999X & 15.6 & 86.2 \\
\hline Local Seed Company & LS4889XS & 15.1 & 91.4 \\
\hline Hubner & H46-29R2X & 15.8 & 75.0 \\
\hline Hubner & H49-27R2X & 15.9 & 87.1 \\
\hline Credenz & CZ 4869X & 15.6 & 75.7 \\
\hline Credenz & CZ 4979X & 15.2 & 81.4 \\
\hline USG & 7447XTS & 15.2 & 73.6 \\
\hline USG & 7480XT & 15.8 & 68.9 \\
\hline LG Seed & C4227RX & 15.7 & 77.7 \\
\hline LG Seed & C4845RX & 15.9 & 88.4 \\
\hline Pioneer & P42A96X & 15.6 & 75.3 \\
\hline Pioneer & P48A60X & 14.9 & 92.3 \\
\hline Progeny & P 4821 RX & 14.9 & 83.4 \\
\hline NK Seed & S44-C7X & 15.4 & 80.7 \\
\hline NK Seed & S42-B9XS & 15.5 & 78.9 \\
\hline Asgrow & AG47X9 & 15.0 & 86.2 \\
\hline
\end{tabular}




\begin{tabular}{|l|l|c|c|} 
Asgrow & AG48X9 & 15.2 & 88.2 \\
\hline Channel & 4519 R2X/SR & 15.1 & 79.0 \\
\hline Channel & 4218 R2X/SR & 15.0 & 78.3 \\
\hline MorSoy & MS 4616 RXT & 15.3 & 77.1 \\
\hline & & & \\
\hline & AVERAGE & $\mathbf{1 5 . 4}$ & $\mathbf{8 2 . 4}$ \\
\hline
\end{tabular}

Discussion: Use these data, as well as other test plot results, when making variety selections. 
2020 PRINCE GEORGE COUNTY MATURITY GROUP 4 SOYBEAN COMPARISONS

Cooperators:

Previous Crop:

Soil Type:

Tillage:

Planting Date:

Seeding Rate/Row Spacing:

Fertilization:

Crop Protection:

Harvest Date:

Harvest Equipment:

Brand

CHECK

Asgrow

Asgrow

Pioneer

Pioneer

USG

USG

Hubner

Hubner

Dyna-Gro

Dyna-Gro

Progeny

NK Seed

NK Seed

Credenz

Credenz

LG Seed

LG Seed

Local Seed Company

Local Seed Company

MorSoy

MorSoy

CHECK

Producer:

Extension:

Sean Finney

Scott Reiter

Wheat

Aycock and Montross silt loam

No-till

June 15, 2020

220,000 seed/acre, 7.5 inch rows

120-50-120 to wheat

Roundup 1 qt/A + XtendiMax $22 \mathrm{oz} / \mathrm{A}$

November 29, 2020

John Deere 9510 + weigh wagon

\begin{tabular}{|l|c|c|}
\hline Variety & Moisture\% & Yield (bu/A) \\
\hline Hubner 51-10R2X & 17.3 & 58.2 \\
\hline AG47X9 & 16.7 & 62.1 \\
\hline AG48X9 & 16.5 & 58.7 \\
\hline P42A96X & 17.1 & 53.4 \\
\hline P48A60X & 16.7 & 61.0 \\
\hline 7447XTS & 16.5 & 60.1 \\
\hline 7480XT & 16.4 & 53.9 \\
\hline H46-29R2X & 16.1 & 59.7 \\
\hline H49-27R2X & 16.6 & 58.6 \\
\hline S48XT56 & 16.5 & 57.0 \\
\hline S48XT90 & 16.1 & 55.5 \\
\hline P 4821 RX & 15.6 & 53.0 \\
\hline S42-B9XS & 16.8 & 57.1 \\
\hline S44-C7X & 16.9 & 55.3 \\
\hline CZ 4869X & 16.5 & 60.5 \\
\hline CZ 4979X & 16.5 & 53.5 \\
\hline C4845RX & 16.4 & 50.7 \\
\hline C4227RX & 16.8 & 50.1 \\
\hline LS4889XS & 16.1 & 56.2 \\
\hline LS4999X & 15.9 & 54.6 \\
\hline MS 4616 RXT & 15.6 & 59.3 \\
\hline MS 4846 RXT & 16.0 & 52.0 \\
\hline Hubner 51-10R2X & 16.1 & 65.0 \\
\hline & & $\mathbf{5 6 . 8}$ \\
\hline AVERAGE & $\mathbf{1 6 . 4}$ & \\
\hline
\end{tabular}


Discussion: This was a great yielding double-crop soybean trial. Excess water was an issue as it rained for a week after planting. The last three weeks of July were hot and dry. August and September provided about 20 inches of total rainfall. Test weights ranged from 54.9 to $56.7 \mathrm{lbs} / \mathrm{bu}$ with an average of $56 \mathrm{lbs} / \mathrm{bu}$. Credenz CZ4869X, Credenz CZ4979X, Local Seed LS4889XS, and MorSoy MS4616RXT were taller varieties with notable lodging but were still harvestable. 
2020 SOUTHAMPTON COUNTY MATURITY GROUP 4 SOYBEAN COMPARISONS

\section{Cooperators:}

Previous Crop:

Soil Type:

Tillage:

Planting Date:

Seeding Rate/Row Spacing:

Fertilization:

Crop Protection:

Harvest Date:

Harvest Equipment:
Producer:

Extension:

\begin{tabular}{|l|l|c|c|}
\hline Brand & Variety & Moisture\% & Yield (bu/A) \\
\hline Asgrow & AG47X9 & 13.2 & 44.9 \\
\hline Asgrow & AG48X9 & 13.6 & 44.5 \\
\hline Pioneer & P42A96X & 13.1 & 41.3 \\
\hline Pioneer & P48A60X & 13.8 & 52.3 \\
\hline USG & 7447XTS & 13.3 & 50.9 \\
\hline USG & 7480XT & 13.2 & 54.0 \\
\hline Hubner & H46-29R2X & 13.1 & 46.2 \\
\hline Hubner & H49-27R2X & 13.4 & 50.3 \\
\hline Dyna-Gro & S48XT56 & 13.8 & 48.7 \\
\hline Dyna-Gro & S48XT90 & 13.7 & 50.2 \\
\hline Progeny & P 4821 RX & 13.7 & 44.4 \\
\hline NK Seed & S42-B9XS & 13.1 & 50.3 \\
\hline NK Seed & S44-C7X & 13.4 & 44.7 \\
\hline Credenz & CZ 4869X & 13.8 & 54.1 \\
\hline Credenz & CZ 4979X & 13.8 & 54.3 \\
\hline LG Seed & C4845RX & 13.5 & 44.3 \\
\hline LG Seed & C4227RX & 13.6 & 39.9 \\
\hline Local Seed Company & LS4889XS & 13.8 & 54.2 \\
\hline Local Seed Company & LS4999X & 13.9 & 44.5 \\
\hline MorSoy & MS 4616 RXT & 13.2 & 44.9 \\
\hline MorSoy & MS 4846 RXT & 13.5 & 45.8 \\
\hline & AVERAGE & $\mathbf{1 3 . 5}$ & $\mathbf{4 7 . 8}$ \\
\hline
\end{tabular}

Discussion: Planting conditions were wet early on, followed by extremely hot/dry conditions for all of

July. Yields remained favorable due to rainfall events in August.
Pittman Farms LLC

Josh Holland, VCE Southampton

Peanuts

Emporia fine sandy loam

No-till

May 26, 2020

$155,000 / 15$ " rows

5-13-43@275 lbs./Acre

Pre: RoundUp@ $32 \mathrm{oz}+$ Valor @ $2 \mathrm{oz}$

Post: RoundUp@32 oz + Xtendimax @ 28 oz

November 24, 2020

John Deere 9450 w/ 918 Header 
Cooperators:

Previous Crop:

Soil Type:

Tillage:

Planting Date:

Seeding Rate/Row Spacing:

Fertilization:

Crop Protection:
Producer: $\quad$ F.F. Chandler, Jr. and Louis Chandler

Extension: Stephanie Romelczyk, ANR - Westmoreland

Trent Jones, ANR - Northumberland/Lancaster

Corn

Kempsville loam

No-till

May 11, 2020

$132,000 / 30$ " rows

20-50-75-5S

Preplant: Gramoxone 2 pts/A + Liberate 3.4 pts/A + Broadaxe 24 oz/A

Postemergence:

1. Makaze $1.5 \mathrm{qts} / \mathrm{A}+$ Weather Gard $1 \mathrm{qt} / 100 \mathrm{gal}+$ Anthem Max 3 oz/A + Radiate 2 oz/A

2. Makaze $1 \mathrm{qt} / \mathrm{A}+$ Miravis Top $13.7 \mathrm{oz} / \mathrm{A}+$ Sniper Helios 6 oz/A + Radiate 2 oz/A + Maximum Npact K $1 \mathrm{gal} / \mathrm{A}$

November 6, 2020

John Deere 9400

Harvest Equipment:

\begin{tabular}{|l|l|c|c|}
\hline Brand & Variety & Moisture\% & Yield (bu/A) \\
\hline MorSoy & MS 4846 RXT & 13.7 & 80.5 \\
\hline MorSoy & MS 4616 RXT & 13.9 & 74.9 \\
\hline Credenz & CZ 4979X & 13.6 & 77.6 \\
\hline Credenz & CZ 4869X & 13.8 & 71.9 \\
\hline Local Seed Company & LS4889XS & 14.0 & 74.7 \\
\hline Local Seed Company & LS4999X & 13.2 & 74.2 \\
\hline NK Seed & S44-C7X & 13.4 & 70.0 \\
\hline NK Seed & S42-B9XS & 13.8 & 70.1 \\
\hline Progeny & P 4821 RX & 13.7 & 77.0 \\
\hline Dyna-Gro & S48XT90 & 13.3 & 75.4 \\
\hline Dyna-Gro & S48XT56 & 12.9 & 80.0 \\
\hline LG Seed & C4227RX & 13.7 & 69.3 \\
\hline LG Seed & C4845RX & 13.6 & 76.3 \\
\hline Hubner & H49-27R2X & 13.5 & 52.7 \\
\hline Hubner & H46-29R2X & 13.2 & 68.8 \\
\hline USG & 7480XT & 13.6 & 75.4 \\
\hline USG & 7447XTS & 13.5 & 70.9 \\
\hline Asgrow & AG47X9 & 13.2 & 73.0 \\
\hline
\end{tabular}




\begin{tabular}{|l|l|l|l|} 
Asgrow & AG48X9 & 13.2 & 70.1 \\
\hline Pioneer & P48A60X & 12.9 & 67.3 \\
\hline & & & \\
\hline & AVERAGE & $\mathbf{1 3 . 5}$ & $\mathbf{7 2 . 5}$ \\
\hline
\end{tabular}

Discussion: Given the year, soybean yields were good. Pioneer 42A96X was not harvested as part of the variety trial. Local Seed Company LS4889XS had lodging issues at harvest. 


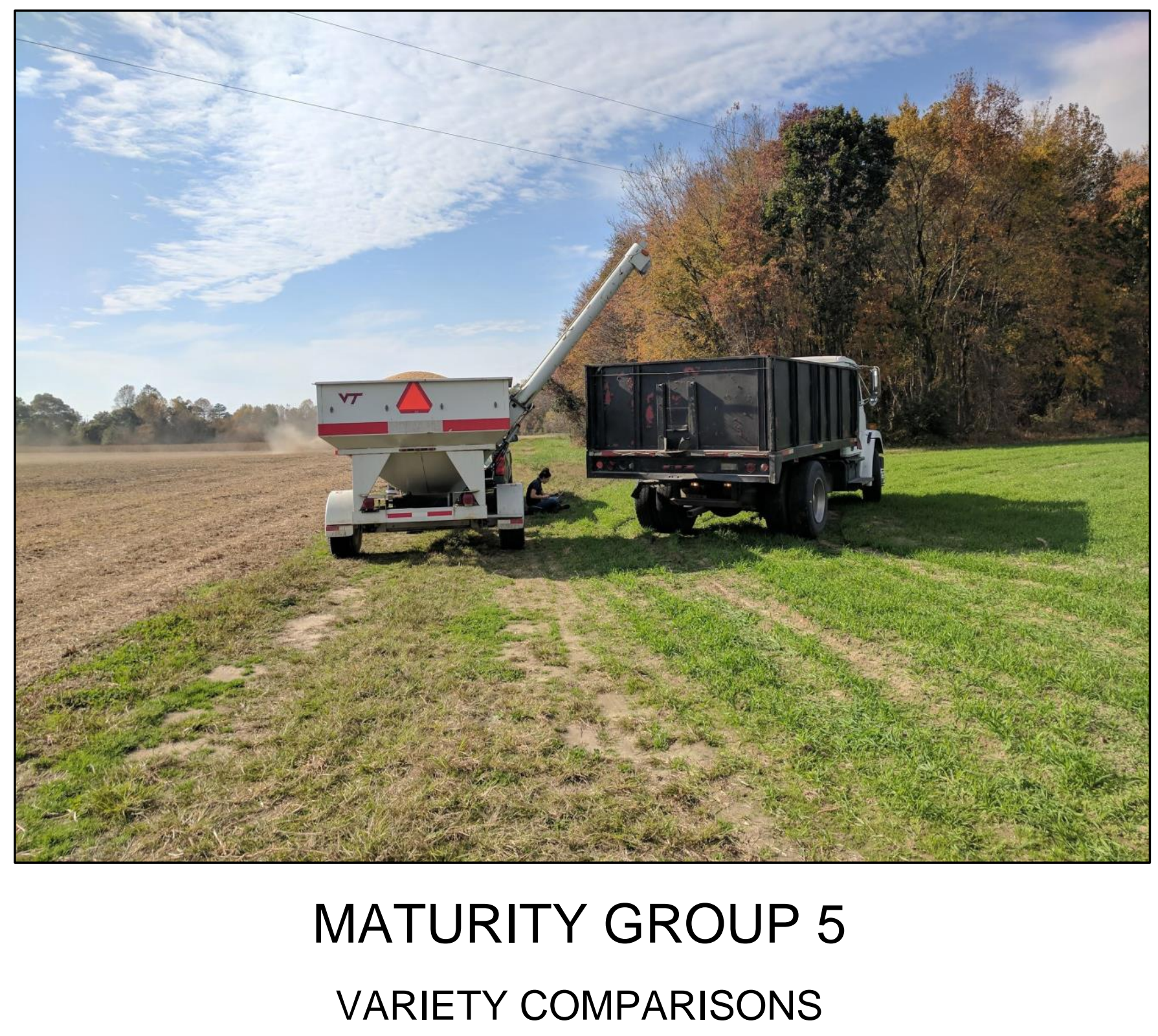


10

동

음

 р|әу시

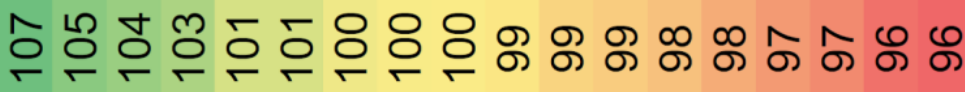

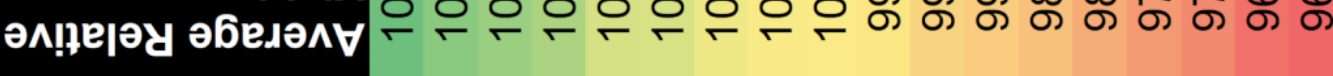

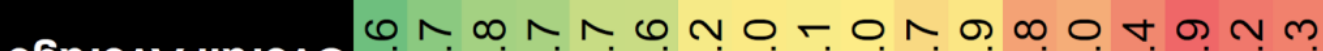
ช

\section{puejuәqunyдon}

の

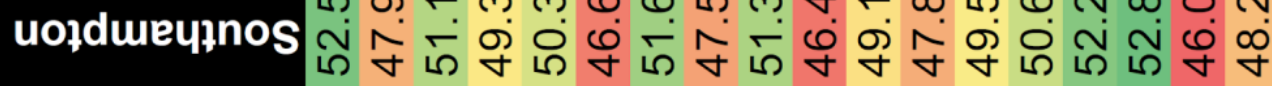

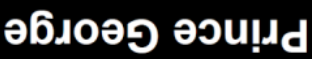

$\infty$ n $\infty$ L ภ $\operatorname{odx} \mathbf{6} \forall \forall \Lambda$

$\sim$ -

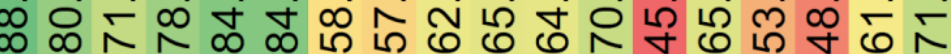

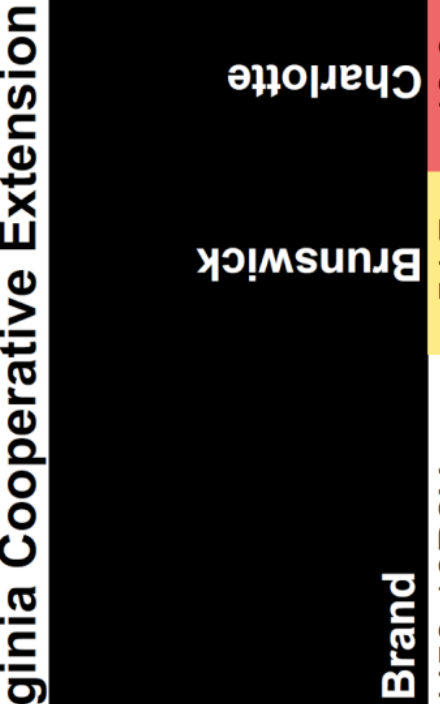

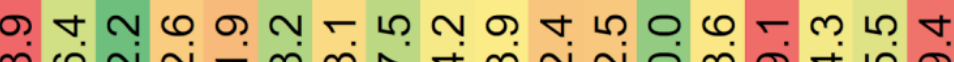

6 ә!pp!mu!a is 虽

Nの ர்

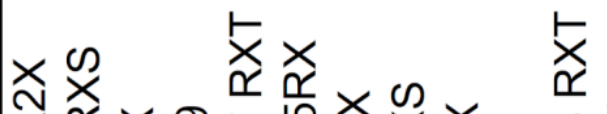

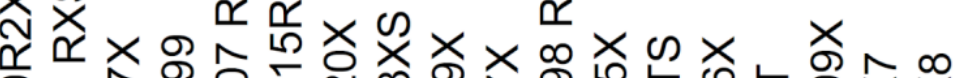
1 0 ○ ט

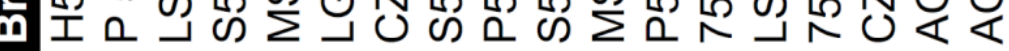

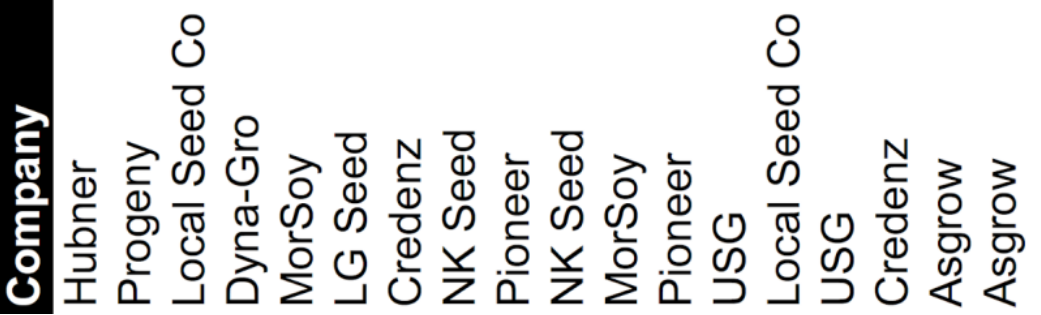

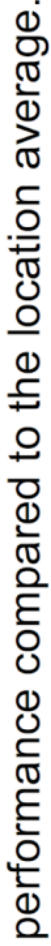

$\stackrel{2}{\longleftarrow} \dot{0}$

¿

ஸे

ช

ก

กิ

$\stackrel{\oplus}{\stackrel{\oplus}{\rightleftarrows}}$

진

을

ธั่ ัे

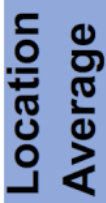

을 을

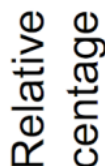

(1) ำ

రิ

is ত্র

$\stackrel{ \pm}{\text { 은 }} \underset{*}{*} \pm$ 
Cooperators:

Previous Crop:

Soil Type:

Tillage:

Planting Date:

Seeding Rate/Row Spacing:

Fertilization:

Crop Protection:

Harvest Date:

Harvest Equipment:
Producer:

Extension:

Edward, William and Howard Wright

Taylor Clarke

Lindy Fimon

Sarah Rutherford

Tobacco followed by wheat for grain

Appling-Mattaponi complex

No-till in Wheat Stubble

June 26, 2020

200,000 on 15 " rows

30-70-90 to wheat

Burndown: Liberty + Volunteer

POST: Roundup and Flexstar

November 18, 2020

Gleaner R42

\begin{tabular}{|l|l|c|c|}
\hline Brand & Variety & Moisture\% & Yield (bu/A) \\
\hline CHECK & Pioneer 55A49X & 11.9 & 55.5 \\
\hline MorSoy & MS 5607 RXT & 12.1 & 46.0 \\
\hline MorSoy & MS 5398 RXT & 11.8 & 44.5 \\
\hline Local Seed Company & LS5386X & 11.7 & 35.8 \\
\hline Local Seed Company & LS5087X & 11.9 & 61.8 \\
\hline CHECK & Pioneer 55A49X & 11.6 & 46.5 \\
\hline Asgrow & AG55X7 & 11.9 & 43.1 \\
\hline Asgrow & AG56X8 & 11.6 & 39.2 \\
\hline Pioneer & P52A05X & 11.4 & 43.5 \\
\hline Pioneer & P55A49X & 11.7 & 48.6 \\
\hline USG & 7529XTS & 11.9 & 55.7 \\
\hline USG & 7540XT & 11.9 & 54.6 \\
\hline Hubner & H50-10R2X & 11.8 & 54.7 \\
\hline Dyna-Gro & S56XT99 & 12.0 & 53.3 \\
\hline Progeny & P 5016 RXS & 11.4 & 54.9 \\
\hline CHECK & Pioneer 55A49X & 11.3 & 54.8 \\
\hline NK Seed & S51-R3XS & 11.6 & 61.4 \\
\hline NK Seed & S53-F7X & 11.9 & 59.5 \\
\hline Credenz & CZ 5299X & 12.0 & 59.9 \\
\hline Credenz & CZ 5420X & 12.4 & 58.0 \\
\hline LG Seed & LGS5315RX & 12.5 & 53.6 \\
\hline Local Seed Company & LS5087X & 12.8 & 56.1 \\
\hline
\end{tabular}




\begin{tabular}{|l|l|c|c|} 
Local Seed Company & LS5386X & 12.9 & 55.7 \\
\hline MorSoy & MS 5398 RXT & 12.8 & 55.4 \\
\hline MorSoy & MS 5607 RXT & 12.3 & 49.1 \\
\hline CHECK & Pioneer 55A49X & 11.9 & 50.0 \\
\hline & & & \\
\hline \multicolumn{2}{|r|}{ AVERAGE } & $\mathbf{1 2 . 0}$ & $\mathbf{5 2 . 0}$ \\
\hline
\end{tabular}

Discussion: Use these data, as well as other test plot results, when making variety selections. 


\section{CHARLOTTE COUNTY MATURITY GROUP 5 SOYBEAN COMPARISONS}

Cooperators:

Producer: $\quad$ Grind-N-Stone Farm

Extension: Joanne Jones - Charlotte County

Bruce Jones - Appomattox County

Previous Crop:

Soil Type:

Tillage:

wheat hay

Appling fine sandy loam

No-till

Planting Date:

May 26, 2020

Seeding Rate/Row Spacing:

15 inch - John Deere 7000 140,000 seed per acre

Fertilization:

Crop Protection:

$0-30-60$

Burndown: Glyphosate + Dicamba

POST: Glyphosate + Engenia + Province insecticide 1 month after planting

Harvest Date:

November 11, 2020

Harvest Equipment:

Gleaner R52

\begin{tabular}{|l|l|c|c|}
\hline Brand & Variety & Moisture\% & Yield (bu/A) \\
\hline MorSoy & MS 5398 RXT & 14.8 & 52.4 \\
\hline Dyna-Gro & S56XT99 & 14.4 & 52.6 \\
\hline Pioneer & P52A05X & 14.7 & 52.5 \\
\hline Credenz & CZ 5299X & 14.1 & 54.3 \\
\hline MorSoy & MS 5607 RXT & 14.1 & 51.9 \\
\hline USG & 7529XTS & 15.0 & 60.0 \\
\hline Credenz & CZ 5420X & 14.8 & 53.1 \\
\hline Local Seed Company & LS5386X & 15.0 & 53.6 \\
\hline Progeny & P 5016 RXS & 14.9 & 56.4 \\
\hline USG & 7540XT & 15.1 & 49.1 \\
\hline Pioneer & P55A49X & 14.8 & 54.2 \\
\hline NK Seed & S53-F7X & 15.1 & 53.9 \\
\hline Asgrow & AG55X7 & 14.6 & 55.5 \\
\hline NK Seed & S51-R3XS & 14.6 & 57.5 \\
\hline Local Seed Company & LS5087X & 15.1 & 62.2 \\
\hline LG Seed & LGS5315RX & 15.2 & 58.2 \\
\hline Hubner & H50-10R2X & 14.7 & 48.9 \\
\hline Asgrow & AG56X8 & 14.1 & 49.4 \\
\hline & & & \\
\hline & AVERAGE & $\mathbf{1 4 . 7}$ & $\mathbf{5 4 . 2}$ \\
\hline
\end{tabular}

Discussion: Use these data, as well as other test plot results, when making variety selections. 


\section{DINWIDDIE COUNTY MATURITY GROUP 5 SOYBEAN COMPARISONS}

Cooperators:

Previous Crop:

Soil Type:

Tillage:

Planting Date:

Seeding Rate/Row Spacing:

Fertilization:

Crop Protection:
Producer: Billy Bain

Extension: Mike Parrish

Double Crop Soybeans - cover crop was Guardian Rye

Mattaponi sandy loam

No-till

May 26, 2020

$160,000 / 30$ in rows

Pre-plant 300lbs 5-10-30

Burn down - 1qt Roundup + 1pt Dual $+24 \mathrm{oz}$ Barrage + $1 \mathrm{qt} / 10080 / 20$

POST - \#1 = 12 oz Engenia + Clasp drift retardant + 1qt Roundup + 1qt/100 80/20

$\# 2$ = 1qt Roundup + 1qt 80/20 + 8 oz Besiege

November 11, 2020

2588 Case International

\section{Harvest Equipment:}

\begin{tabular}{|l|l|c|c|}
\hline Brand & Variety & Moisture\% & Yield (bu/A) \\
\hline CHECK & Local LC5588X & 12.2 & 61.5 \\
\hline Asgrow & AG55X7 & 12.2 & 58.6 \\
\hline Asgrow & AG56X8 & 11.3 & 60.1 \\
\hline Pioneer & P52A05X & 12.0 & 58.7 \\
\hline Pioneer & P55A49X & 12.0 & 59.0 \\
\hline USG & 7529XTS & 11.8 & 57.4 \\
\hline USG & 7540XT & 12.0 & 58.2 \\
\hline Hubner & H50-10R2X & 12.0 & 59.6 \\
\hline Dyna-Gro & S56XT99 & 11.8 & 58.6 \\
\hline CHECK & Local LC5588X & 11.4 & 56.8 \\
\hline Progeny & P 5016 RXS & 11.7 & 59.2 \\
\hline NK Seed & S51-R3XS & 12.2 & 55.7 \\
\hline NK Seed & S53-F7X & 11.9 & 57.3 \\
\hline Credenz & CZ 5299X & 11.3 & 48.9 \\
\hline Credenz & CZ 5420X & 11.9 & 59.1 \\
\hline LG Seed & LGS5315RX & 11.9 & 52.3 \\
\hline Local Seed Company & LS5087X & 11.9 & 53.5 \\
\hline Local Seed Company & LS5386X & 11.2 & 52.2 \\
\hline MorSoy & MS 5398 RXT & 12.0 & 62.6 \\
\hline MorSoy & MS 5607 RXT & 12.0 & 58.2 \\
\hline CHECK & Local LC5588X & 11.5 & 55.8 \\
\hline
\end{tabular}




\begin{tabular}{|l|c|c|c|} 
& & & \\
\hline & AVERAGE & $\mathbf{1 1 . 8}$ & $\mathbf{5 7 . 3}$ \\
\hline
\end{tabular}

Discussion: Use these data, as well as other test plot results, when making variety selections. 
2020 NORTHUMBERLAND COUNTY MATURITY GROUP 5 SOYBEAN COMPARISONS

Cooperators:

Previous Crop:

Soil Type:

Tillage:

Planting Date:

Seeding Rate/Row Spacing:

Fertilization:

Crop Protection:
Producer:

Extension:

\section{Industry:}

Harvest Date:

Harvest Equipment:
Bleak House Farm

Trent Jones, David Holshouser

Ian Walker, Pioneer Seeds

Corn

Woodstown fine sandy loam

No-till

May 4, 2020

128,000 Seed / Acre - 15" Row Spacing

May 4 - 2 gal. 3-18-18, 1 qt. Mn in furrow

April 7 - 16 oz. Dicamba, 48 oz.

Roundup PowerMax, 3 oz. Envive, 1 qt.

Prowl H2O

July 7 - 32 oz. Roundup, 12.8 oz.

Engenia, 2 qt. Task Force 3D

July 28 - 8 oz. Besiege, 13.7 oz. Miravis

Top, 2 qt. Maximum N-Pact K

November 9, 2020

Case 8250 with Mac Don FD135

\begin{tabular}{|l|l|c|c|}
\hline Brand & Variety & Moisture\% & Yield (bu/A) \\
\hline Asgrow & AG56X8 & 14.8 & 84.4 \\
\hline Asgrow & AG55X7 & 14.1 & 61.2 \\
\hline MorSoy & MS 5607 RXT & 14.7 & 84.1 \\
\hline MorSoy & MS 5398 RXT & 14.3 & 64.2 \\
\hline Dyna-Gro & S56XT99 & 14.6 & 78.5 \\
\hline USG & 7529XTS & 15.0 & 45.4 \\
\hline USG & 7540XT & 15.0 & 53.2 \\
\hline Progeny & P 5016 RX & 15.0 & 80.1 \\
\hline Local Seed Company & LS5386X & 14.8 & 65.9 \\
\hline Local Seed Company & LS5087X & 14.4 & 71.1 \\
\hline Pioneer & P55A49X & 14.9 & 62.9 \\
\hline Pioneer & P52A05X & 14.6 & 70.2 \\
\hline LG Seed & LGS5315RX & 14.8 & 84.0 \\
\hline Hubner & H50-10R2X & 14.7 & 88.7 \\
\hline NK Seed & S51-R3XS & 15.1 & 57.2 \\
\hline NK Seed & S53-F7X & 14.7 & 65.1 \\
\hline Credenz & CZ 5420X & 14.8 & 58.3 \\
\hline Credenz & CZ 5299X & 15.0 & 48.4 \\
\hline Asgrow & AG56X8 & 14.7 & 58.8 \\
\hline
\end{tabular}




\begin{tabular}{|l|l|l|l|} 
& & & \\
\hline & AVERAGE & 14.7 & 67.5 \\
\hline
\end{tabular}

Discussion: Use these data, as well as other test plot results, when making variety selections. 


\section{PRINCE GEORGE COUNTY MATURITY GROUP 5 SOYBEAN COMPARISONS}

Cooperators:

Previous Crop:

Soil Type:

Tillage:

Planting Date:

Seeding Rate/Row Spacing:

Fertilization:

Crop Protection:

Harvest Date:

Harvest Equipment:
Producer:

Extension:

\begin{tabular}{|l|l|c|c|}
\hline Brand & Variety & Moisture\% & Yield (bu/A) \\
\hline CHECK & Hubner 51-10R2X & 16.1 & 65.0 \\
\hline Asgrow & AG55X7 & 15.5 & 60.5 \\
\hline Asgrow & AG56X8 & 15.0 & 57.3 \\
\hline Pioneer & P52A05X & 15.0 & 62.6 \\
\hline Pioneer & P55A49X & 15.6 & 60.8 \\
\hline USG & 7529XTS & 16.0 & 60.8 \\
\hline USG & 7540XT & 15.9 & 59.1 \\
\hline Hubner & H50-10R2X & 15.9 & 59.5 \\
\hline Dyna-Gro & S56XT99 & 15.4 & 59.8 \\
\hline Progeny & P 5016 RXS & 15.3 & 59.6 \\
\hline NK Seed & S51-R3XS & 15.7 & 56.5 \\
\hline NK Seed & S53-F7X & 15.5 & 53.8 \\
\hline Credenz & CZ 5299X & 15.7 & 58.9 \\
\hline Credenz & CZ 5420X & 15.9 & 57.0 \\
\hline LG Seed & LGS5315RX & 15.8 & 51.1 \\
\hline Local Seed Company & LS5087X & 15.7 & 58.9 \\
\hline Local Seed Company & LS5386X & 15.7 & 51.9 \\
\hline MorSoy & MS 5398 RXT & 15.7 & 50.5 \\
\hline MorSoy & MS 5607 RXT & 15.2 & 52.5 \\
\hline CHECK & Hubner 51-10R2X & 15.6 & 52.3 \\
\hline & AVERAGE & $\mathbf{1 5 . 6}$ & $\mathbf{5 7 . 4}$ \\
\hline
\end{tabular}

Discussion: This was a great yielding double-crop soybean trial. Excess water was an issue as it rained 1.5 inches the night after planting. The last three weeks of July were hot and dry. Test weights ranged from 55.5 to $56.9 \mathrm{lbs} / \mathrm{bu}$ with an average of $56.2 \mathrm{lbs} / \mathrm{bu}$. USG7540XT and Credenz CZ5420X had notable lodging but were still harvestable. The last six plots had some drowned spots that likely affected yields. With 30 inches of rain from August 1 - November 30 it did not take much depression for water to pool this season. 


\section{SOUTHAMPTON COUNTY MATURITY GROUP 5 SOYBEAN COMPARISONS}

Cooperators:

Previous Crop:

Soil Type:

Tillage:

Planting Date:

Seeding Rate/Row Spacing:

Fertilization:

Crop Protection:
Producer:

Extension:

Harvest Date:

Harvest Equipment:
Pittman Farms LLC

Josh Holland, VCE Southampton

Peanuts

Emporia fine sandy loam

No-till

May 26, 2020

$155,000 / 15$ " rows

5-13-43@ 275 lbs./Acre

Pre: RoundUp@32 oz.+Valor@ 2 oz

Post: RoundUp@32 oz.+Xtendimax @ 28 oz

November 24, 2020

John Deere 9450 w/ 918 Header

\begin{tabular}{|l|l|c|c|}
\hline Brand & Variety & Moisture\% & Yield (bu/A) \\
\hline Asgrow & AG55X7 & 13.6 & 46.0 \\
\hline Asgrow & AG56X8 & 13.8 & 48.2 \\
\hline Pioneer & P52A05X & 13.3 & 47.8 \\
\hline Pioneer & P55A49X & 13.2 & 51.3 \\
\hline USG & 7529XTS & 13.1 & 49.5 \\
\hline USG & 7540XT & 13.4 & 52.2 \\
\hline Hubner & H50-10R2X & 13.4 & 52.5 \\
\hline Dyna-Gro & S56XT99 & 13.6 & 49.3 \\
\hline Progeny & P 5016 RX & 13.0 & 47.9 \\
\hline NK Seed & S51-R3XS & 13.1 & 47.5 \\
\hline NK Seed & S53-F7X & 13.3 & 46.4 \\
\hline Credenz & CZ 5299X & 13.1 & 52.8 \\
\hline Credenz & CZ 5420X & 13.2 & 51.6 \\
\hline LG Seed & LGS5315RX & 13.5 & 46.6 \\
\hline Local Seed Company & LS5087X & 13.1 & 51.1 \\
\hline Local Seed Company & LS5386X & 13.4 & 50.6 \\
\hline MorSoy & MS 5398 RXT & 13.4 & 49.1 \\
\hline MorSoy & MS 5607 RXT & 13.2 & 50.3 \\
\hline & & & \\
\hline & \multicolumn{1}{|c|}{ AVERAGE } & $\mathbf{1 3 . 3}$ & $\mathbf{4 9 . 5}$ \\
\hline
\end{tabular}

Discussion: Planting conditions were wet early on, followed by extremely hot/dry conditions for all of July. Yields remained favorable due to rainfall events in August. 


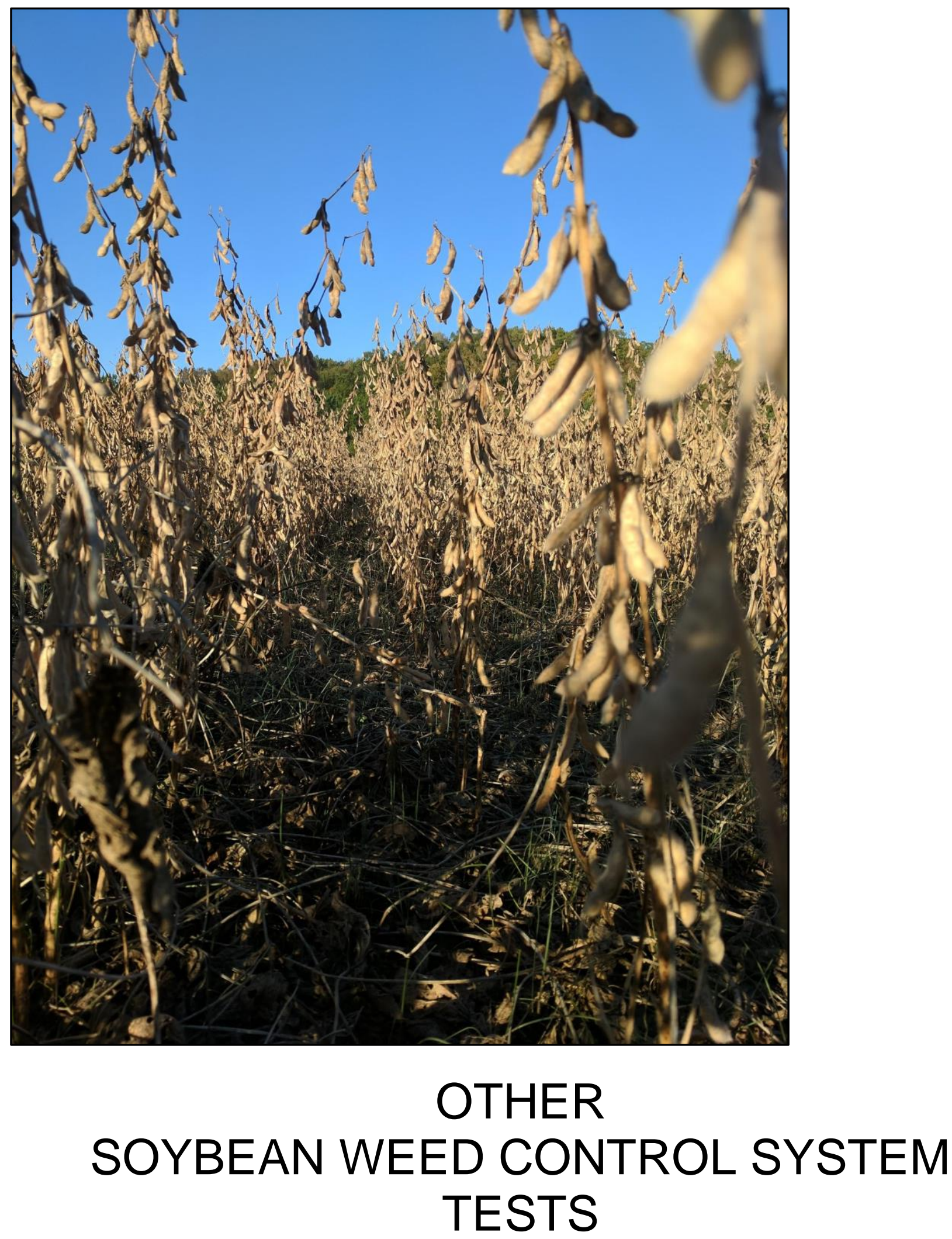




\section{Virginia Cooperative Extension On-farm Soybean Variety Trials - Liberty Link}

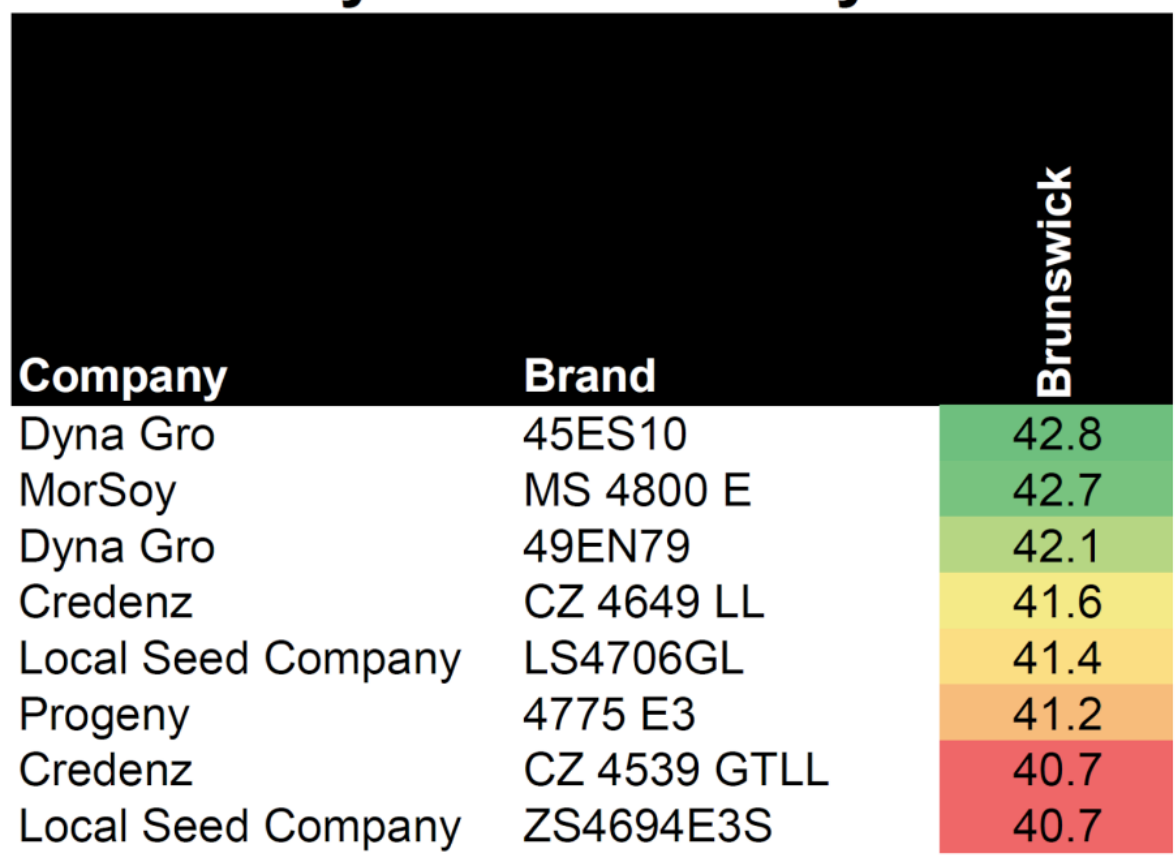

\section{LOCATION AVERAGE

MorSoy

Credenz

Credenz

Progeny

Dyna Gro

Local Seed Company

Dyna Gro
MS $5110 \mathrm{E}$

CZ 5147 LL

CZ 5859 LL

$5211 \mathrm{E} 3$

52LL66

ZS5098E3

55LS75

LOCATION

AVERAGE
44.9

43.2

41.6

40.9

39.4

38.7

38.1

41.0 


\section{BRUNSWICK COUNTY LIBERTY LINK SOYBEAN COMPARISONS}

\section{Cooperators:}

Previous Crop:

Soil Type:

Tillage:

Planting Date:

Seeding Rate/Row Spacing:

Fertilization:

Crop Protection:

\section{Producer:}

Extension:
William and Howard Wright

Taylor Clarke

Lindy Fimon

Sara Rutherford

Wheat

Appling-Mattaponi complex

No-till

June 23, 2020

200,000 on 15 " rows

30-70-80 at wheat planting

Burndown: Roundup (1 qt), 2-4D (10 oz), Envive (3 oz)

POST: Liberty (1 qt), Volunteer (8 oz)

November 23, 2020

Gleaner R42

\section{Harvest Equipment:}

Harvest Date:

\begin{tabular}{|l|l|c|c|}
\hline Brand & \multicolumn{1}{|c|}{ Variety } & Moisture\% & Yield (bu/A) \\
\hline Southern Harvest & SH5120LL & 15.2 & 34.2 \\
\hline Southern Harvest & SH4817LL & 15.2 & 38.6 \\
\hline Southern Harvest & SH5515LL & 15.1 & 39.9 \\
\hline Credenz & CZ 4539 GTLL & 14.9 & 40.7 \\
\hline Credenz & CZ 4649 LL & 15.0 & 41.6 \\
\hline Credenz & CZ 5147 LL & 13.9 & 43.2 \\
\hline Credenz & CZ 5859 LL & 13.7 & 41.6 \\
\hline Progeny & P 4775 E3S & 14.5 & 41.2 \\
\hline Progeny & P 5211 E3 & 14.5 & 40.9 \\
\hline Southern Harvest & SH5515LL & 14.5 & 42.8 \\
\hline Dyna-Gro & S45ES10 & 14.5 & 42.8 \\
\hline Dyna-Gro & S49EN79 & 14.6 & 42.1 \\
\hline Dyna-Gro & S52LL66 & 14.7 & 39.4 \\
\hline Dyna-Gro & S55LS75 & 14.2 & 38.1 \\
\hline MorSoy & MS 4800 E & 14.9 & 42.7 \\
\hline MorSoy & MS 5110 E & 15.3 & 44.9 \\
\hline Southern Harvest & SH5515LL & 15.3 & 40.1 \\
\hline Local Seed Company & ZS4694E3S & 15.2 & 40.7 \\
\hline Local Seed Company & LS4706GL & 15.0 & 41.4 \\
\hline Local Seed Company & ZS5098E3S & 15.5 & 38.7 \\
\hline Southern Harvest & SH4817LL & 15.5 & 24.1 \\
\hline
\end{tabular}




\begin{tabular}{|l|l|l|l|} 
Southern Harvest & SH5215LL & 15.0 & 37.1 \\
\hline Southern Harvest & SH5515LL & 15.5 & 34.8 \\
\hline & & & \\
\hline & AVERAGE & $\mathbf{1 4 . 9}$ & $\mathbf{3 9 . 6}$ \\
\hline
\end{tabular}

Discussion: Two varieties exhibited noticeable deer preferential grazing: Southern Harvest 4817LL and Local Seed Company ZS5098E3S. 


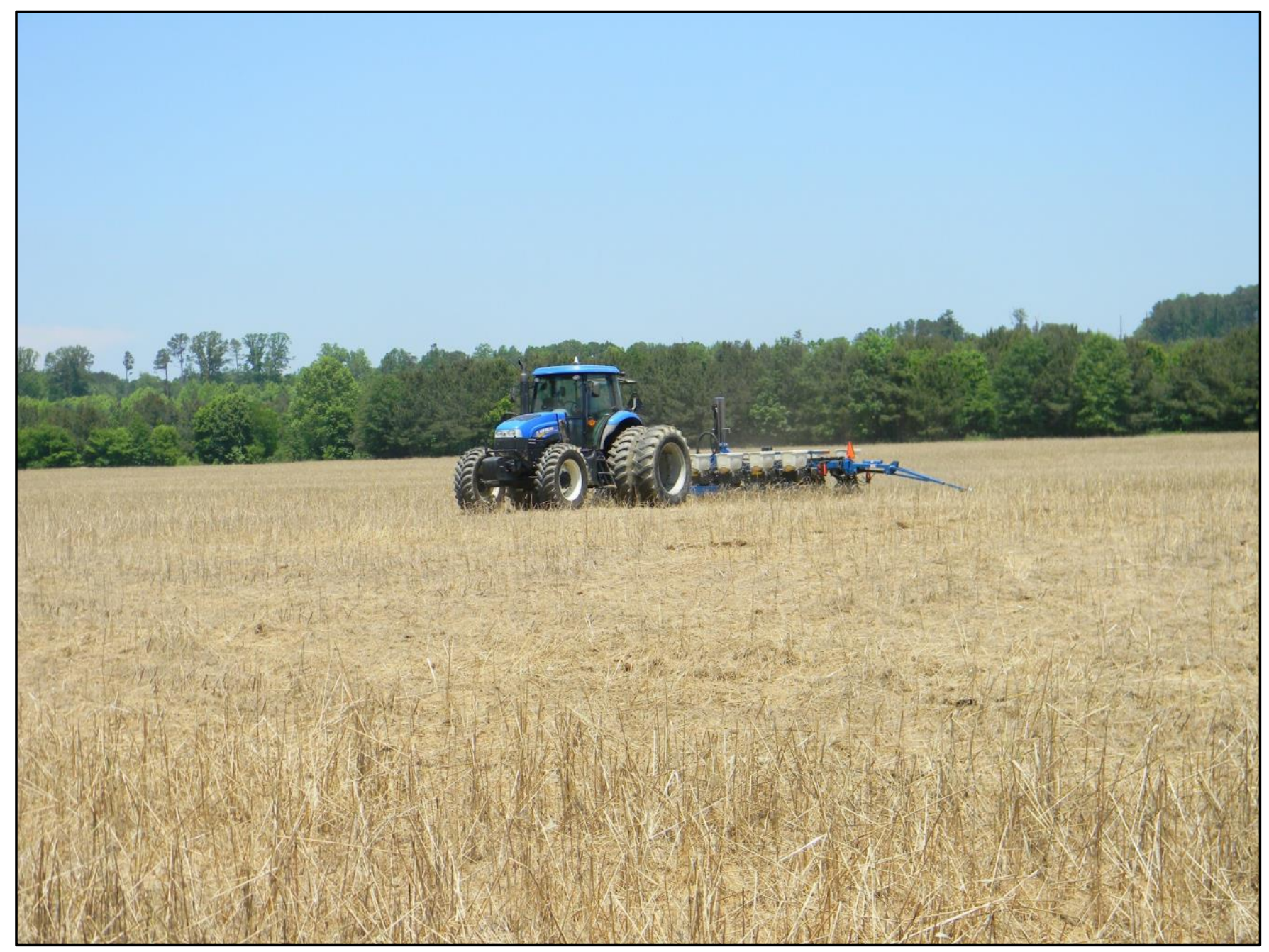

\section{Other Research}




\section{NORTHUMBERLAND COUNTY MATURITY GROUP 2.5 - 3.9 SOYBEAN COMPARISONS}

Cooperators:

Previous Crop:

Soil Type:

Tillage:

Planting Date:

Seeding Rate/Row Spacing:

Fertilization:

Crop Protection:

\author{
Producer: $\quad$ Bleak House Farm \\ Extension: Trent Jones, David Holshouser \\ Industry: $\quad$ Ian Walker, Pioneer Seeds \\ Corn \\ Woodstown fine sandy loam \\ No-till
}

May 4, 2020

128,000 Seed / Acre - 15" Row Spacing

May 4 - 2 gal. 3-18-18, 1 qt. Mn in furrow April 7 - 16 oz. Dicamba, 48 oz. Roundup PowerMax, 3 oz. Envive, 1 qt. Prowl H2O July 7 - 32 oz. Roundup, 12.8 oz. Engenia, 2 qt. Task Force 3D

July 28 - 8 oz. Besiege, 13.7 oz. Miravis Top, 2 qt. Maximum N-Pact K

November 9, 2020

Case 8250 with Mac Don FD135

\section{Harvest Equipment:}

\begin{tabular}{|l|l|c|c|}
\hline Brand & Variety & Moisture\% & Yield (bu/A) \\
\hline Local Seed Company & LS3976X & 15.5 & 81.4 \\
\hline LG Seed & C2888RX & 16.1 & 53.7 \\
\hline LG Seed & LGS3777RX & 16.4 & 93.3 \\
\hline NK Seed & S37-A4X & 16.0 & 75.0 \\
\hline NK Seed & S39-G2X & 15.7 & 95.8 \\
\hline Pioneer & P39A58X & 16.0 & 81.6 \\
\hline Pioneer & P37A69X & 15.2 & 73.4 \\
\hline Dyna-Gro & S37XS89 & 16.2 & 83.7 \\
\hline MorSoy & MS 3907 RXT & 15.5 & 75.2 \\
\hline Asgrow & AG36X6 & 15.8 & 84.4 \\
\hline Asgrow & AG38X8 & 15.2 & 78.3 \\
\hline Channel & 3919 R2X & 15.7 & 75.8 \\
\hline Local Seed Company & LS3976X & 15.4 & \\
\hline & & & $\mathbf{7 9 . 4}$ \\
\hline & AVERAGE & $\mathbf{1 5 . 7}$ & \\
\hline
\end{tabular}

Discussion: Use these data, as well as other test plot results, when making variety selections. 


\section{WESTMORELAND COUNTY SOYBEAN FOLLOWING COVER CROP STUDY}

Cooperators:

Previous Crop:

Soil Type:

Tillage:

Planting Date:

Variety:

Seeding Rate:

Fertilization:

Crop Protection:

Harvest Date:

Harvest Equipment:
Producer: Keith Balderson

Extension: Robbie Longest, ANR - Essex County \&

Stephanie Romelczyk, ANR - Westmoreland

Other: Danny Withers, Three Rivers SWCD

Corn followed by cover crops or left fallow

Kempsville loam and Montross silt loam

Continuous no-till

October 16, 2019 for Cover Crops and May 15, 2020 for

Soybeans

Asgrow 43XRR2X

approx. 125,000 seeds/A

16-78-75 per acre

Burndown: Roundup + Sharpen

Pre-emergence: Envive

Post-emergence: Makaze and Synchrony

November 7, 2020

John Deere 7720 w/18 foot header

\begin{tabular}{|l|c|c|}
\hline Treatment & Moisture\% & Yield (bu/A) \\
\hline Rye & 14.2 & 64.4 \\
\hline Wheat & 14.1 & 63.5 \\
\hline Barley & 14.3 & 63.9 \\
\hline Oats & 14.4 & 62.4 \\
\hline Fallow & 14.0 & 61.9 \\
\hline
\end{tabular}

Discussion: The purpose of this plot was to evaluate the performance of full-season soybean following small grain cover crops and fallow land (corn residue.) Barley, oats, rye, and wheat cover crops were established on October 16, 2019 following corn harvest using a no-till drill. A very good stand of all four species was achieved, but the germination on the rye seed was only about $50 \%$, which resulted in a thinner rye stand than desired. Bio-mass samples were taken by cutting all plant material from two 1 square foot samples in each species on April 10th. Samples were air-dried for several days until the samples were crispy and bio-mass was calculated on a dry matter per acre basis. As expected, the rye cover crop produced the most bio-mass. The results are reported below. 
Bio-mass (April 10, 2020)

\begin{tabular}{|l|l|}
\hline Species & Weight (Lbs. per acre) \\
\hline Barley & 4,764 \\
\hline Oats & 4,764 \\
\hline Rye & 7,487 \\
\hline Wheat & 6,125 \\
\hline
\end{tabular}

Cover crops were terminated using herbicides in mid-April. Full-season soybeans were planted with a no-till drill on May 15th. A good stand of soybeans was obtained in all treatments. After a dry spell from June $25^{\text {th }}$ to July $21^{\text {st }}$, growing conditions were very good. Harvest was somewhat delayed due to damp conditions, but seed quality was very good and no excessive shattering losses were noted. Overall yields were very good in all treatments. This was only a demonstration plot so no hard conclusions should be made from the results. We encourage farmers to continue to experiment with cover crops to help them determine how they can fit into their cropping systems.

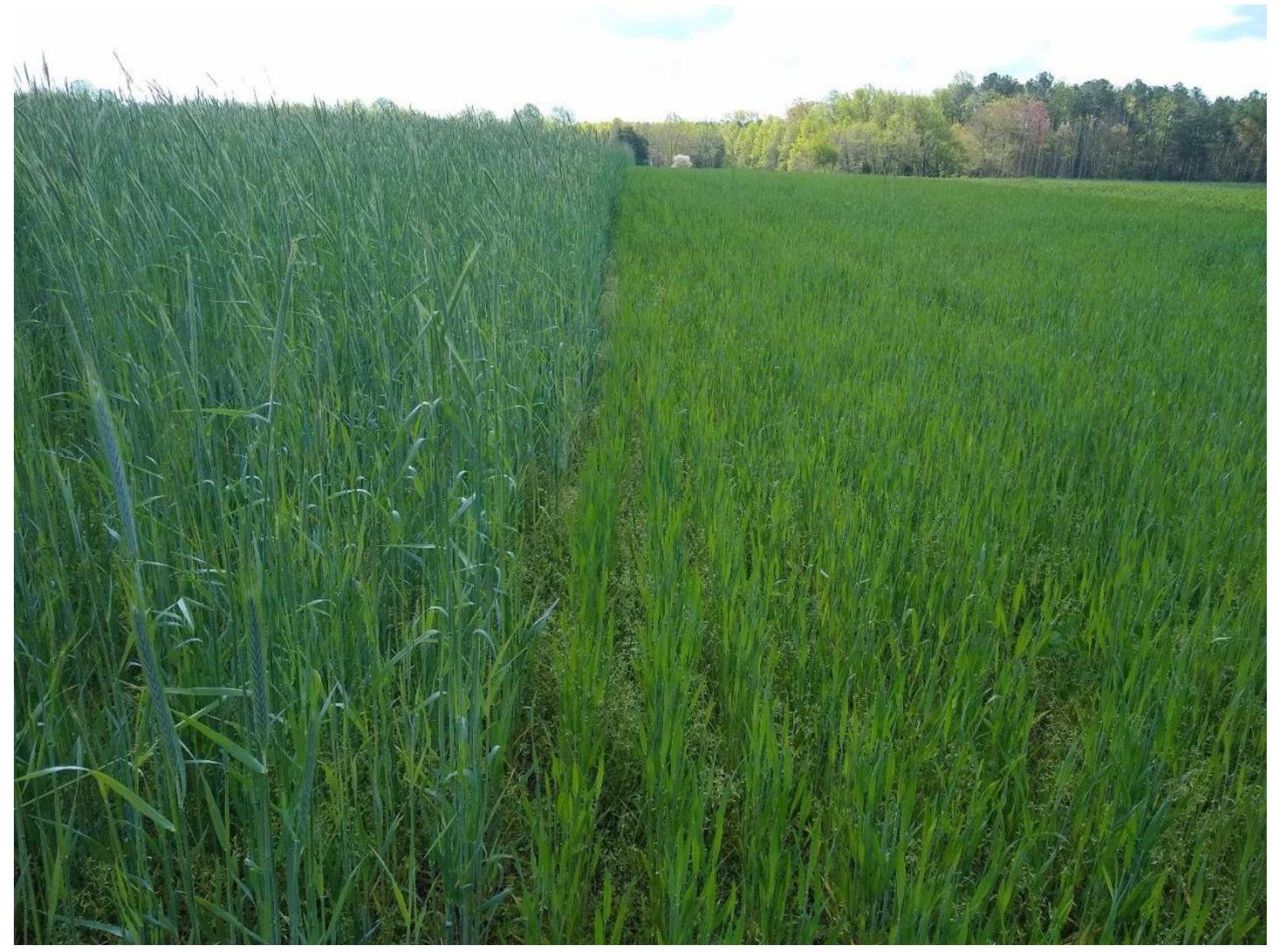

Figure 1. View of rye and wheat cover crops prior to burndown in April 2020 


\section{ESSEX COUNTY PLENISH SOYBEAN EVALUATION STUDY}

Cooperators:

Previous Crop:

Soil Type:

Tillage:

Planting Date:

Seeding Rate:

Fertilization:

Crop Protection:

Harvest Date:

Harvest Equipment:
Producer:

Extension:

Industry:

\begin{tabular}{|l|c|c|c|}
\hline & Moisture\% & $\begin{array}{c}\text { Test } \\
\text { Weight } \\
\text { (lbs/bu) }\end{array}$ & Yield (bu/A) \\
\hline Pioneer 41T65PR & 13.4 & 57.0 & 49.3 \\
\hline Pioneer 42T71PR & 12.9 & 59.0 & 54.6 \\
\hline Pioneer 46A45PR & 13.6 & 59.2 & 59.3 \\
\hline Pioneer 48A94PR & 13.7 & 60.0 & 65.9 \\
\hline & & & \\
\hline AVERAGE & $\mathbf{1 3 . 4}$ & $\mathbf{5 8 . 8}$ & $\mathbf{5 7 . 3}$ \\
\hline
\end{tabular}

*Please note that these varieties were not replicated at this location

Discussion: The purpose of this plot was to evaluate four Pioneer brand Plenish soybean varieties onfarm in a comparison plot. The varieties ranged in maturity from 4.1 to 4.8 . Good yields were observed in this plot despite a hot and dry period during the growing season in June and July. Test weights ranged from 57-60 lbs/bu. Samples were submitted to Perdue Agribusiness in Tappahannock for oil content testing, but results were not available at the time of this report.

These varieties are marketed as having high oleic soybean oil content. There has been advertisement from some grain elevator locations offering a premium program for high oleic soybeans, but this offer varies by location and not all grain elevators are currently offering premiums or a program. Please contact your local grain elevator representative for more information about this program.

More extensive, replicated research is needed to draw conclusions from this data, but it is presented here for demonstration purposes. 


\section{ESSEX COUNTY BRASSICA COVER CROP SOYBEAN DEMONSTRATION}

Cooperators:

Previous Crop:

Soil Type:

Tillage:

Planting Date:

Variety:

Seeding Rate/Row

Spacing:

Fertilization:

Crop Protection:
Producer: Brandon Farms

Extension: Robbie Longest, VCE-Essex

Other: $\quad$ Keith Balderson, NRCS

Danny Withers, Three Rivers SWCD

Corn

Pamunkey

No-till

Cover Crops: October 16, 2019

Soybeans: May 12, 2020

Mission Early MG4

Cover Crop: Tillage radish (9 lb/A), Rapeseed (7 lb/A)

Soybeans: 140,000 seeds/A (15-inch rows)

0-35-75 (May 6, 2020)

Cover Crop Burndown: (April 8, 2020)

$1 \mathrm{qt} / \mathrm{A}$ Roundup (51\%) + 0.5 pt/A 2,4-D

Post-Emergent (Soybeans):

$1 \mathrm{qt} /$ A Roundup (51\%)

November 8, 2020

John Deere S670 w/ 625F Header

Harvest Equipment:$$
\text { w } 625 \mathrm{~F} \text { Header }
$$

\begin{tabular}{|l|c|c|c|}
\hline Treatment & Replication & Moisture\% & Yield (bu/A) \\
\hline Tillage Radish & 1 & 14.5 & 67.1 \\
\hline Check (Fallow) & 1 & 14.5 & 70.6 \\
\hline Tillage Radish & 2 & 14.6 & 68.5 \\
\hline Rapeseed & 1 & 14.3 & 70.2 \\
\hline Check (Fallow) & 2 & 15.3 & 68.2 \\
\hline Tillage Radish & 3 & 14.8 & 68.7 \\
\hline & & & $\mathbf{6 8 . 1}$ \\
\hline Average Tillage Radish* & & $\mathbf{1 4 . 6}$ & $\mathbf{6 9 . 4}$ \\
\hline Average Check (Fallow)* & & $\mathbf{1 4 . 9}$ & $\mathbf{7 0 . 2}$ \\
\hline Rapeseed* & & $\mathbf{1 4 . 3}$ & $\mathbf{6}$
\end{tabular}

* Please note that due to weather-related flooding at harvest, only the above plots were able to be harvested for yield data. At planting, each treatment was replicated 4 times, for a total of 12 plots, but only half could be harvested. Not all treatments had the same number of replications harvested to be averaged. 
Discussion: The purpose of this demonstration plot was to evaluate the nutrient cycling and deposition of two brassica cover crop species, tillage radish and rapeseed, when compared to a fallow check plot. Cover crops were planted following corn and prior to a soybean crop. Soybean yield following these cover crop treatments was not the primary objective of this study, but partial yield data is reported above. Three replications were planned for all treatments, but some replications could not be harvested due to poor field conditions at harvest.

Cover crops were drilled October 16, 2019 and terminated on April 8, 2020. Neither the tillage radish nor rapeseed plots winter-killed and were still living at the time of herbicide termination. Soybeans were planted May 12, 2020. Baseline soil samples were taken from all 12 plots on October 29, 2019 and soil pH, $\mathrm{P}$, and $\mathrm{K}$ were measured using the Virginia Tech Soil Testing Lab. Soil samples were taken again from each of the 12 plots on May 4 and June 30, 2020 to a soil depth of 6 inches. Soil nitrate tests (PSNT) were also collected on June 30, 2020 to a soil depth of 12 inches. Soil sampling interval results from each plot were averaged by treatment and are reported in the table below (Table composed and provided courtesy of Bob Waring).

\begin{tabular}{|c|c|c|c|c|}
\hline Treatment ID & $\frac{\text { Sampling }}{\text { Date }}$ & $\frac{P}{(\mathrm{lbs} / \mathrm{ac})}$ & $\begin{array}{c}\underline{K} \\
\text { (lbs/ac) }\end{array}$ & Notes \\
\hline \multirow[t]{6}{*}{ Check Avg. } & Oct 29, 2019 & 22 & 128 & \multirow{5}{*}{ Difference } \\
\hline & May 4, 2020 & 23 & 130 & \\
\hline & \multirow{4}{*}{$\begin{array}{l}\text { Oct } 29,2019 \\
\text { June } 30,2020\end{array}$} & 1 & 2 & \\
\hline & & 22 & 128 & \\
\hline & & 33 & 174 & \\
\hline & & 11 & 46 & Check \\
\hline \multirow[t]{8}{*}{ Tillage Radish Avg. } & Oct 29, 2019 & 24 & 117 & \multirow{5}{*}{ Difference } \\
\hline & May 4, 2020 & 25 & 127 & \\
\hline & & 1 & 10 & \\
\hline & Oct 29, 2019 & 24 & 117 & \\
\hline & \multirow[t]{4}{*}{ June 30, 2020} & 29 & 220 & \\
\hline & & 5 & 103 & \multirow{3}{*}{$\begin{array}{c}\text { Difference } \\
\text { Check } \\
\text { Treatment minus Check }\end{array}$} \\
\hline & & 11 & 46 & \\
\hline & & 6 & 57 & \\
\hline \multirow[t]{8}{*}{ Rapeseed Avg. } & Oct 29, 2019 & 27 & 119 & \multirow{8}{*}{$\begin{array}{c}\text { Difference } \\
\text { Check } \\
\text { Treatment minus Check }\end{array}$} \\
\hline & May 4, 2020 & 27 & 123 & \\
\hline & & 0 & 4 & \\
\hline & Oct 29, 2019 & 27 & 119 & \\
\hline & \multirow[t]{4}{*}{ June 30,2020} & 39 & 156 & \\
\hline & & 12 & 37 & \\
\hline & & 11 & 46 & \\
\hline & & 1 & 9 & \\
\hline
\end{tabular}


The table above reports treatment averages for each of the sampling dates and compares the average baseline soil test reading taken just after planting of the cover crop treatments (October 29, 2019) to the treatment average soil test results taken prior to soybean planting (May 4, 2020) and in-season (June 30, 2020). This table expresses the changes in soil test readings for $P$ and $K$ for the three treatments. Since the entire plot received commercial fertilizer, the "value" for that fertilizer was determined in the fallow treatment (check) and then used to theoretically adjust the values for the tillage radish and rapeseed averages to determine a net increase (gain) or decrease (loss) in $\mathrm{P}$ and $\mathrm{K}$. It is interesting to note that it appears that the tillage radish treatment resulted in an increase of $57 \mathrm{lbs} / \mathrm{A}$ of $\mathrm{K}$ in the soil test result measured on June 30. This could potentially be explained by the taproot growth of this species that may have been able to pull $\mathrm{K}$ from lower in the soil profile and recycle those nutrients after decay.

More research is needed to justify the effect that these cover crop species may have on nutrient cycling of phosphorus and potassium. In theory, these species are taking up phosphorus and potassium from the soil profile during growth and releasing these nutrients back into the soil during the decomposition process. However, the rate at which these species decay and their nutrient uptake potential and cycling ability should be evaluated further in Virginia. 
2020 NORTHUMBERLAND DOUBLE-CROP SOYBEAN SEEDING RATE STUDY

$\begin{array}{lll}\text { Cooperators: } & \text { Producer: } & \text { Harris Farms, Inc } \\ & \text { Extension: } & \text { Lindsey Bowers, Virginia Tech } \\ & & \text { Dr. David Holshouser, Virginia Tech } \\ & & \text { Trent Jones, Lancaster/Northumberland } \\ \text { Previous Crop: } & \text { Wheat } \\ \text { Planting Date: } & \text { June 30, 2020 } \\ \text { Tillage: } & \text { No-till } \\ \text { Variety: } & \text { Mission A4847NSXR2 } \\ \text { Row Spacing: } & 20^{\prime \prime} \\ \text { Harvest Date: } & & \text { November 17, 2020 }\end{array}$

\begin{tabular}{|c|c|c|c|c|}
\hline Treatment (Seeding Rate/A) & Replication & Moisture\% & Yield (bu/A) & Average NDVI \\
\hline 170,000 & 1 & 12.7 & 50.0 & 0.79 \\
\hline 210,000 & 1 & 12.9 & 51.0 & 0.78 \\
\hline 170,000 & 2 & 12.6 & 52.0 & 0.77 \\
\hline 210,000 & 2 & 13.3 & 53.8 & 0.78 \\
\hline 170,000 & 3 & 12.7 & 61.9 & 0.76 \\
\hline 210,000 & 3 & 13.0 & 60.2 & 0.77 \\
\hline 170,000 & 4 & 13.1 & 61.2 & 0.77 \\
\hline 210,000 & 4 & 13.5 & 59.2 & 0.77 \\
\hline \multicolumn{2}{|c|}{ AVERAGE $(\mathbf{1 7 0 , 0 0 0 )}$} & 12.8 & 56.3 & 0.77 \\
\hline \multicolumn{2}{|c|}{ AVERAGE $(210,000)$} & 13.2 & 56.0 & 0.78 \\
\hline \multicolumn{2}{|c|}{ LSD $P=0.05$} & 0.25 & 2.2 & 0.01 \\
\hline
\end{tabular}

\section{Discussion:}

Seed is one of the most expensive costs that soybean farmers incur. This experiment evaluated two seeding rates, the farmer's current practice of 170,000 seed/acre and one with 40,000 more seed. Normal difference vegetative index (NDVI) measurements, which are a good indication of plant growth, were taken every two weeks at 100 -foot intervals within each seeding rate strip beginning at early plant growth. There was little difference in growth averaged across all replications. In comparison to the greater seeding rate, the 170,000 seed/acre rate yielded 0.3 bushels/acre more, but this difference was not significant. This validates previous theories that less seed can be used if growth from the lower seeding rate is not compromised. Even though the yield difference was not significant, this small gain in yield represents an additional \$3.30 increase in income at $\$ 11.00$ per bushel. Furthermore, using 40,000 less seeds/acre resulted in an additional $\$ 14.29$ gain in seed savings, assuming an average seed cost of $\$ 50 /$ unit. This resulted in a benefit of $\$ 17.60$ per acre from using less seed. In this experiment, there was no benefit to using the greater seeding rate. Nonetheless, the farmer may choose to use the higher seeding rate to avoid risk of yield loss, especially if the field is not as productive as this field. 


\section{NORTHUMBERLAND COUNTY FULL-SEASON SOYBEAN SEEDING RATE TRIAL}

$\begin{array}{lll}\text { Cooperators: } & \text { Producer: } & \text { Giese Farm } \\ & \text { Extension: } & \text { Lindsey Bowers, Virginia Tech } \\ & & \text { Dr. David Holshouser, Virginia Tech } \\ & \text { Trent Jones, Lancaster/Northumberland } \\ \text { Previous Crop: } & \text { Corn } \\ \text { Planting Date: } & \text { June 4, 2020 } \\ \text { Tillage: } & \text { No-till } \\ \text { Variety: } & \text { Pioneer P46A57BX } \\ \text { Row Spacing: } & 15 " \\ \text { Harvest Date: } & \text { November 10, 2020 }\end{array}$

\begin{tabular}{|c|c|c|c|c|}
\hline Treatment (Seeding Rate/A) & Replication & Moisture\% & Yield (bu/A) & Average NDVI \\
\hline 135,000 & 1 & 15.2 & 53.2 & 0.82 \\
\hline 95,000 & 1 & 16.1 & 49.7 & 0.82 \\
\hline 135,000 & 2 & 14.9 & 54.1 & 0.83 \\
\hline 95,000 & 2 & 14.5 & 49.3 & 0.82 \\
\hline 95,000 & 3 & 14.8 & 50.9 & 0.80 \\
\hline 135,000 & 3 & 14.5 & 52.6 & 0.81 \\
\hline 135,000 & 4 & 14.4 & 54.5 & 0.81 \\
\hline 95,000 & 4 & 14.1 & 52.8 & 0.81 \\
\hline \multicolumn{2}{|c|}{ AVERAGE $(95,000)$} & 14.9 & $\mathbf{5 0 . 7}$ & 0.81 \\
\hline \multicolumn{2}{|c|}{ AVERAGE $(\mathbf{1 3 5 , 0 0 0 )}$} & 14.8 & 53.6 & 0.82 \\
\hline \multicolumn{2}{|r|}{ LSD P=0.05 } & 0.7 & 1.8 & 0.01 \\
\hline
\end{tabular}

\section{Discussion:}

Seed is one of the most expensive costs that soybean farmers incur. This experiment evaluated two seeding rates, the farmer's current practice of 135,000 seed/acre and one with 40,000 less seed. Normal difference vegetative index (NDVI) measurements, which are a good indication of plant growth, were taken every two weeks at 100 -foot intervals within each seeding rate strip beginning at early plant growth. Average growth was slightly better at the greater seeding rate compared to the lesser rate (as indicated by NDVI). The 95,000 seed/acre rate yielded 2.9 bushels/acre less than the 135,000 seed/acre rate. This gain in yield over the lower seeding rate represents a $\$ 31.90$ per acre increase in income. Although decreasing the seeding rate by 40,000 seeds/acre results in a $\$ 14.29$ savings, assuming an average seed cost of $\$ 50 /$ unit, this less-than-optimal seeding rate resulted in a net loss of $\$ 17.61$. Therefore, there was little benefit to using less seed. To avoid risk of yield loss, farmers may want to use greater seeding rates when planting in early-June. 


$\begin{array}{lll}\text { Cooperators: } & \text { Producer: } & \text { Bleak House Farm } \\ & \text { Extension: } & \text { Lindsey Bowers, Virginia Tech } \\ & \text { Dr. David Holshouser, Virginia Tech } \\ & \text { Trent Jones, Lancaster/Northumberland } \\ & \text { Soybean } \\ \text { Previous Crop: } & \text { No-till } \\ \text { Tillage: } & \text { May 4, 2020 } \\ \text { Planting Date: } & \text { Asgrow AG48X9 } \\ \text { Variety: } & 15 " \text { row spacing } \\ \text { Seeding Rate/Row Spacing: } & \text { November 9, 2020 } \\ \text { Harvest Date: } & \end{array}$

\begin{tabular}{|c|c|c|c|c|}
\hline Treatment (Seeding Rate/A) & Replication & Moisture\% & Yield (bu/A) & Average NDVI \\
\hline 125,000 & 1 & 18.0 & 65.7 & 0.85 \\
\hline 85,000 & 1 & 17.8 & 65.9 & 0.83 \\
\hline 125,000 & 2 & 17.6 & 66.0 & 0.85 \\
\hline 85,000 & 2 & 17.4 & 63.4 & 0.82 \\
\hline 125,000 & 3 & 17.2 & 66.3 & 0.85 \\
\hline 85,000 & 3 & 17.1 & 63.6 & 0.81 \\
\hline 125,000 & 4 & 16.8 & 69.4 & 0.85 \\
\hline 85,000 & 4 & 16.7 & 66.7 & 0.83 \\
\hline \multicolumn{2}{|c|}{ AVERAGE $(85,000)$} & 17.3 & 64.9 & 0.82 \\
\hline \multicolumn{2}{|c|}{ AVERAGE $(\mathbf{1 2 5 , 0 0 0 )}$} & 17.4 & 66.9 & 0.85 \\
\hline \multicolumn{2}{|c|}{ LSD P $=0.05$} & 0.07 & 1.70 & 0.01 \\
\hline
\end{tabular}

\section{Discussion:}

Seed is one of the most expensive costs that soybean farmers incur. This experiment evaluated two seeding rates, the farmer's current practice of 125,000 seed/acre and one with 40,000 less seed. Normal difference vegetative index (NDVI) measurements, a good indication of plant growth, were taken every two weeks at 100 -foot intervals within each seeding rate strip beginning at early plant growth. Growth was better with the greater seeding rate (as indicated by NDVI) and the 125,000 seed/acre rate yielded 2.0 bushels/acre more than the 85,000 seed/acre rate. This gain in yield over the lower seeding rate represents a $\$ 22.00$ increase in income at $\$ 11.00$ per bushel. Decreasing the seeding rate by 40,000 seeds/acre results in a $\$ 14.29$ savings, assuming an average seed cost of $\$ 50 /$ unit. Therefore, in this experiment, there appears to be a nearly $\$ 8 /$ acre benefit to using the greater seeding rate. This and past research indicate that to avoid risk of yield loss, soybean farmers should not use seeding rates less than 90,000 . 
2020 SUFFOLK LATE-PLANTED SOYBEAN SEEDING RATE STUDY

$\begin{array}{lll}\text { Cooperators: } & \text { Producer: } & \text { Mike Ellis, Suffolk } \\ & \text { Extension: } & \text { Lindsey Bowers, Virginia Tech } \\ & \text { Dr. David Holshouser, Virginia Tech } \\ \text { Previous Crop: } & & \text { Cotton } \\ \text { Planting Date: } & \text { June 30, 2020 } \\ \text { Tillage: } & \text { No-till } \\ \text { Variety: } & \text { Hubner H59-18R2X } \\ \text { Row Spacing: } & 15 " \\ \text { Harvest Date: } & & \text { November 20, 2020 }\end{array}$

\begin{tabular}{|c|c|c|c|c|}
\hline Treatment (Seeding Rate/A) & Replication & Moisture\% & Yield (bu/A) & Average NDVI \\
\hline 160,000 & 1 & 12.7 & 48.9 & 0.87 \\
\hline 200,000 & 1 & 12.7 & 52.7 & 0.86 \\
\hline 160,000 & 2 & 12.0 & 47.0 & 0.85 \\
\hline 200,000 & 2 & 12.0 & 49.9 & 0.84 \\
\hline 160,000 & 3 & 12.5 & 46.7 & 0.87 \\
\hline 200,000 & 3 & 12.3 & 52.7 & 0.87 \\
\hline AVERAGE (160,000) & $\mathbf{1 2 . 4}$ & $\mathbf{4 7 . 5}$ & $\mathbf{0 . 8 6}$ \\
\hline AVERAGE (200,000) & $\mathbf{1 2 . 3}$ & $\mathbf{5 1 . 8}$ & $\mathbf{0 . 8 6}$ \\
\hline \multicolumn{2}{|c|}{ LSD P=0.05 } & $\mathbf{0 . 2}$ & $\mathbf{2 . 7}$ & $\mathbf{0 . 0 1}$ \\
\hline
\end{tabular}

\section{Discussion:}

Seed is one of the most expensive costs that soybean farmers incur. This experiment evaluated two seeding rates, the farmer's current practice of 200,000 seed/acre and one with 40,000 less seed. Normal difference vegetative index (NDVI) measurements, which are a good indication of plant growth, were taken every two weeks at 100 -foot intervals within each seeding rate strip beginning at early plant growth. There was no average difference in growth between both rates. However, the $160,000 \mathrm{seed} / \mathrm{acre}$ rate yielded 4.3 bushels/acre less than the 200,000 seed/acre rate. This gain in yield from the greater seeding rate represents a $\$ 47.30 /$ acre gain in income at $\$ 11.00$ per bushel. Decreasing the seeding rate by 40,000 seeds/acre results in a $\$ 14.29$ savings, assuming an average seed cost of $\$ 50 /$ unit. This results in a $\$ 33.01$ per acre benefit to using the greater seeding rate although average growth was similar. To avoid risk of yield loss, farmers should use greater seeding rates when soybeans are planted late.

Visit Virginia Cooperative Extension: ext.vt.edu

Virginia Cooperative Extension programs and employment are open to all, regardless of age, color, disability, gender, gender identity, gender expression, national origin, political affiliation, race, religion, sexual orientation, genetic information, veteran status, or any other basis protected by law. An equal opportunity/affirmative action employer. Issued in furtherance of Cooperative Extension work, Virginia Polytechnic Institute and State University, Virginia State University, and the U.S. Department of Agriculture cooperating. Edwin J. Jones, Director, Virginia Cooperative Extension, Virginia Tech, Blacksburg; M. Ray McKinnie, Administrator, 1890 Extension Program, Virginia State University, Petersburg. 